\title{
REVERBERATIONS:
}

\section{HOW MODERN VIDEO GAME DESIGN RELIES ON AUDIO AND HAPTIC COMMUNICATION TO ENFORCE RHETORICS OF PROCEDURE AND PLAY}

\author{
by \\ Saunder Waterman \\ Bachelor of Arts (Honours), University of King's College, 2018
}

Supervisor: Dr. John Shiga

A Major Research Paper presented to Ryerson University

in partial fulfillment

of the requirements for the degree of

Master of Professional Communication

In the program of

Communications

Toronto, Ontario, Canada, 2020

(C)Waterman, 2020 


\section{Author's Declaration}

I hereby declare that I am the sole author of this MRP. This is a true copy of the MRP, including any required final revisions.

I authorize Ryerson University to lend this MRP to other institutions or individuals for the purpose of scholarly research. I further authorize Ryerson University to reproduce this MRP by photocopying or by other means, in total or in part, at the request of other institutions or individuals for the purpose of scholarly research.

I understand that my MRP may be made electronically available to the public. 


\begin{abstract}
Modern video games are increasingly becoming a more mature and respected form of storytelling and art. The way in which games can interact with players provides those players with a control over their product that is unmatched by literature or cinema. Games communicate with players in many direct and indirect ways. This paper explores how audio and haptic modes of communication are employed by different types of video games to support both elements of gameplay and the themes and rhetoric that a game possesses. Specifically, this paper focuses on how Super Smash Bros. Ultimate and Legend of Zelda: Breath of the Wild express different aspects of control and procedural rhetoric through audio and haptic communication. This paper also takes into account philosophical concepts of play, widening the scope of the paper to consider why video games are an essential pastime to generations of players.
\end{abstract}




\section{Acknowledgements}

I would like to extend my deepest thank you to my supervisor Dr. John Shiga for guiding me through this laborious process. Thank you for always being supportive and giving me great notes and advice.

To the faculty and staff at Ryerson, thank you for work you did for all of us students, especially in the latter half of the year.

To my friends and classmates, I am so grateful to have you in my life. Your friendship alone would have made this entire year worth it.

And to my family — thank you for always being there for me. Rhys, thank you for fact checking. Jordan, thank you for everything. 


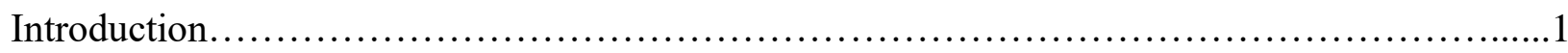

Research Questions.............................................................

Literature Review............................................................4

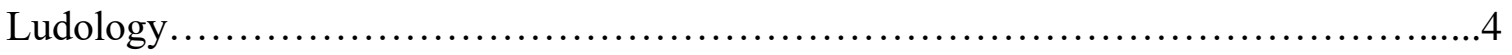

Audio and Haptic Communication...............................................6

Theories of Control.......................................................9

Rhetoric of Video Games..................................................... 11

Research Methods..................................................................... 15

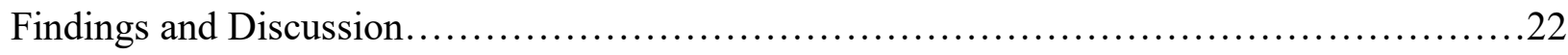

Super Smash Bros. Ultimate - Haptics......................................22

Rhetoric of Control......................................................... 31

Breath of the Wild-Haptics................................................ 35

Breath of the Wild - Audio.................................................. 45

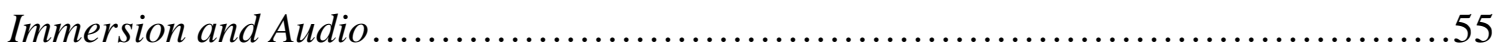

Super Smash Bros. Ultimate - Audio........................................57

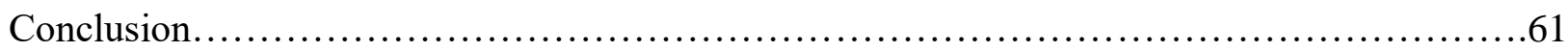




\section{Introduction}

The exponential growth of the video game industry throughout the 21 st century is a significant development in how millions of people worldwide spend their leisure time. Per Newzoo (2019), a gaming and esports analytics company, the global revenue for the video game market was over $\$ 148$ billion in 2019. Statista (2018), a market database company, reported that just shy of 2.4 billion people play video games worldwide. The video game industry has become woven into the fabric of popular culture, specifically online culture. Arguably, the current historical moment in which we find ourselves is a time when video games have reached their apex in popularity. Whether played competitively or recreationally, or whether one tunes in to watch competitive or recreational play, modern video gaming has become a foundational component of popular culture.

What is the allure of video gaming? While video games share many structural elements with film and literature, they have the added benefit of an active, complicit audience that must interact with their product, unlike the movie-goer or bookworm. A video game needs a player, not a watcher or a reader, and that concept of play is crucial to this paper. Elaborating on the philosophical underpinnings of what it means for one to play within the context of a video game will be the first undertaking of this paper. By anchoring this project with a specific play philosophy, this paper endeavours to separate specific games into two different categories for the purpose of comparison. Those two categories will be games that are focused on the 'game' experience of video games, such as competition, and games that focus on the 'play' experience of video games, such as exploration. This distinction between certain video games will be fleshed out in this paper and will set the stage for the primary analysis of this project. 
Video game developers employ a multitude of communication methods to create an immersive, satisfying, and complete experience for their players. An element of this paper will take into consideration different theories of control in video games that are related to concepts like immersion, effectance, and autonomy. Ideas surrounding control are constantly being communicated to the player through the game. While much of this communicative weight is put on the visual and textual mediums of communication, audio and haptic communication play their own significant role in the process. Like other styles of storytelling, specific video game contexts help to support different ideas of rhetoric within the product. Considering how a game communicates with its player will involve discussion surrounding the design intentions of the game and the overall rhetorical message the game conveys. This paper will be analyzing how audio communication (namely music and sound design) and haptic communication (namely the player's relationship to their controller) are manifested within both the gameplay of Legend of Zelda: Breath of the Wild (2017) and Super Smash Bros. Ultimate (2018), and the procedural rhetoric built within these game properties. Video games do employ audio and haptic communication to create rich experiences for the player, but the primary role they play is in the establishment of immersion, effectance and the rhetoric of the game as opposed to simply being elements of aesthetic. Both of these games are major titles recently released by one of the titans of the industry, Nintendo. While the focus of this paper will be on home console gaming specifically, there will be connections and allusions drawn to other areas of gaming, such as PC gaming and mobile gaming. In addition to being examined through the research from my literature review, I will be using insights from my own experience with these games from time to time as well. 


\section{Research Questions}

The research questions I will be addressing throughout this project will be broken into two themes:

Communicating Through Game Design

1) How do video game designers use audio and haptic communication to support elements of gameplay in their game?

2) How does the video game employ audio or haptic communication methods to convey how the game functions and can be played?

3) How do audio and haptic communication methods differ based on the genre or style of the game?

Communicating Game Rhetoric and Messaging:

1) How does the video game employ audio or haptic communication methods to convey the overall themes, morals, and messaging of the game?

2) How do audio and haptic communication methods differ based on the rhetorical nature of the game? 


\section{Literature Review}

The conceptual framework of this project is based on five bodies of literature: (1) the philosophical underpinnings of play and ludology (the study of games, game theory, gaming), (2) audio communication, (3) haptic communication, (4) theories surrounding video game control, and (5) theories of rhetoric commonly employed in video games. Ludology and the philosophical underpinning of play will enable me to identify differences between playing a game for fun and playing a game competitively. These five areas of research are largely disconnected from one another. The literature on ludology is qualitative and theory-based and aims to explore the significance of play in human experience. However, the critical analysis of modern video games and video game culture from the perspective of ludology is not a common area of study. Partnering ludology with audio and haptic-based communication research and video game rhetoric will lead to interesting considerations of how communication is crucial to both the meaning of games and the play experience of games.

\section{Ludology}

Ludology considers play to be a state of being. As is illustrated in Mihaly Csikszentmihalyi's foundational 'flow theory', an individual can achieve a state of complete immersion and proficiency through play (Csikszentmihalyi, 1990). It will be valuable to this project to understand how Csikszentmihalyi thinks communication works within the flow theory. As a state of being, play inherently involves multiple aspects of communication; a player is being communicated to through the parameters of the play experience, and potentially with other players and spectators of the game. According to ludology theorist Brian Sutton-Smith (1997), there are seven different "play rhetorics" that can be used to critically evaluate a state of play (pp. 9-11). This paper extends Sutton-Smith's rhetorics of play to video games and to the process 
of building and maintaining community around video games. Among the seven play rhetorics discussed by Sutton-Smith, the rhetorics of play as the self and as the imaginary appear to be particularly relevant for my project. For Sutton-Smith, both the play rhetorics of self and imaginary are movements of thought rooted in the romantic period, and they are crucially "more concerned with individuals than with groups" (Sutton-Smith, 1997, p. 174). One of the major distinguishing factors between the self and the imaginary is that the self identifies play through the individual's desire, and the imaginary identifies play through the freedom of the individual (Sutton-Smith, 1997, p. 197). These two rhetorics of play have been developed from a Western perspective that focuses on the individualization of the human experience. Play rhetoric of the self endeavors to understand why an individual plays the way they do, and what can be learned from the way in which an individual plays. Rhetoric of the imaginary is a play rhetoric that is closely tied to imaginative people, such as "writers, artists, composers, [and] auteurs", and the variability that play can undertake (Sutton-Smith, 1997, p. 148, 143). Play-as-imaginary is more focused on the structures of play, such as composition and the boundary of the play experience. Both rhetorics acknowledge the ambiguity, unpredictability, and incongruity of play (SuttonSmith, 1997, p. 150, 200), with the imaginary having a larger connection to childlike innocence, and the self being involved with the "passionate and willful character of human play, which involves a willingness, even if a fantasy, to believe in the play venture itself" (p. 198). Csikszentmihalyi and Sutton-Smith's ludology frameworks will be essential components of my project, as they will provide the theoretical framework for understanding the significance of play as a state of being and will act as a lens through which I will analyze all other literature.

Another helpful resource that focuses on the significance of play is Johan Huizinga. In his book, Homo Ludens: A Study of the Play-Element of Culture, Huizinga (1971) argues for a 
recontextualization of what makes us human, saying "Homo Ludens, Man the Player, deserves a place in our nomenclature" (p. 7). Huizinga provides this project with a stimulating philosophical underpinning that positions play as a primary function of how we experience life. Huizinga writes that play, "adorns life, amplifies it and is to that extent a necessity both for the individual - as a life function - and for society by reason of the meaning it contains, its significance, its expressive value, its spiritual and social associations, in short, as a culture function" (Huizinga, 1971, p. 20). Huizinga brings forth a deeper understanding of the play concept, including different ways in which play is active within our lives. One of the most significant ideas Huizinga brings to the table is how communities form around play, and how humans interact with each other through ideas of play.

In addition to its usefulness in analyzing how a state of play is achieved in online video gaming, ludology also raises a philosophical question that is significant to my project. Olli Leino (2019) considers the significance of what he calls 'existential ludology', which specifically relates to the fact that each video game is designed to be a logical, complete world in which the player exists (p. 2). This approach focuses on the role of game designers within the video game industry and whether the current competitive and public environment of how games are consumed changes the way the games ought to be designed. While this question is not at the heart of my research, this does appear to be an underdeveloped area in video game studies, and I anticipate my project will generate insights that relate to this area.

\section{Audio and Haptic Communication}

The research into audio and haptic communication as they relate to video games branches into a few different directions. To begin, it was helpful to consult literature that understood audio and haptic communication as functions of design. Two books which enlightened me on general 
ideas of design were The Design of Everyday Things and Emotional Design, by Don Norman (2002, 2004). These books are staples of design education and provide a general understanding of how haptic and audio modes of communication can influence design. With regards to audio communication, Emotional Design investigates how music and sound can affect a person emotionally (Norman, 2004, pp. 115, 119). The significant contribution from The Design of Everyday Things were Norman's various definitions of important design terminology, such as feedback, mapping, and affordances, which will be useful when considering elements of game design in my project. The book also discusses different ways in which audio and haptic design choices can significantly improve the efficiency of the design in question.

Narrowing the focus from how audio and haptics are used in design to how they are used specifically in video game design, I found literature that focuses on specific game design that incorporates audio or haptic communication in a significant manner. Regarding video game sound design, I used the work of Dr. Karen Collins $(2008,2013)$ to support my argument, namely her books, Game Sound: An Introduction to the History, Theory, and Practice of Video Game Music and Sound Design and Playing with Sound: A Theory of Interacting with Sound and Music in Video Games. While the former is more focused on establishing the context in which we find video game music and sound design today, the latter focuses more on the relationship between sound and player, and how sound is used to connect and immerse the player in the game environment. Beyond these two books, I will be referencing multiple articles that evaluate the use of sound in specific genres or styles of video games, such as horror (Garner, 2013) or audiobased games (Urbanek, 2017), to name a few.

Another important resource focused on audio communication in video games is William Cheng's (2014) Sound Play: Video Games and the Musical Imagination. In his book, Cheng 
examines how five different video games in five different genres use sound and music as a unique game design technique or game activity. Cheng's chapters on Fallout 3, Silent Hill, and Lord of the Rings Online display the utility of audio communication through narrative, immersion, and interactivity, respectively. For Cheng, audio communication is a unique channel in gaming. He writes, "many games grant opportunities to interact with audio phenomena in manners that might not be prudent, practical, or possible in the physical world. In virtual environments, there's unusual license to play with sound, to push the boundaries of its signifying and sensational capabilities in diverse, fantastical contexts. Sound Play lends an ear to how video games give rise to imaginative_ often transgressive — forms of sonic engagement" (Cheng, 2014, p. 4). This resource is one of the major influential texts on this project.

As for literature on haptic communication specifically, beyond the Norman books already mentioned, I focused my research on the kind of control haptic communication provides the player of a game. Matthew Wysocki's (2013) Ctrl-Alt-Play: Essays on Control in Video Gaming touches on exactly this, with specific chapters that directly engage with how control can be achieved through haptic avenues. Video game journalist Mark Brown (2016) conducts research exclusively on video game design, and his informative video on the history of the controller and how controller design affects the types of games that can be made provides a more nuanced understanding of how important haptic communication can be in video games.

One gameplay style that encourages a haptic-based relationship to a game is speedrunning - the drive for proficient, perfected play. An article by James Ash (2012) focuses on the relationship high-level players have with the games they excel at. Using Street Fighter IV as an example, the article explores temporality and how it is experienced by the player through the technology of the video game. While Street Fighter IV is a predominantly visual game, high- 
level players rely on their understanding of the machinations of the game, like frame rate, as well as the rhythm of playing, to achieve their success (Ash, 2012, p. 200). This article speaks to a more haptic understanding of gameplay. In its thesis, it provides new insight to Csikszentmihalyi's flow theory. Ash argues that human experience of temporality is shaped by objects or technologies, whereas flow theory understands the flow state (in which one experiences a sense of timelessness) in terms of the capabilities of a person in carrying out an activity (Ash, 2012, p. 196).

\section{Theories of Control in Video Games}

Communication in video games is intricately tied to ideas of control. There are some studies that focus on the role of the controller within the greater context of video games. Some studies, including Skalski et al. (2011) and Limperos et al. (2011), focus on the design elements of controllers, such as mapping. Both studies compared the control scheme of the innovative Nintendo Wii controller to that of a more traditional controller, like the PlayStation 2 (Limperos et al., 2011). Both studies attempt to evaluate the benefits of natural mapping to feelings of enjoyment, effectance, and interactivity. In video game literature, natural mapping refers to "how closely actions represented in a game match the actions used to bring about that change in a real environment" (Skalski et al., 2011, p. 226). Arcade games typically attempted to provide players with a control scheme that more closely related the actions required within the game. As video game journalist Mark Brown (2016) outlines in his video "Controllers Control Everything", the

arcade game had their own personalized control system built into the game unit itself, whether it be a rolling ball for Marble Madness, twin dials for cooperative play for Pong, or, decades later, a controller shaped as a hunting rifle for games like Big Buck Hunter (Brown, 2016). 
The design of video game controllers can affect the types of games that get made for the controller's console. With the ascension of home consoles, it benefitted console developers to have relative controller uniformity with each other, thus allowing third party game developers to produce a single version of a game that could easily be played on multiple consoles. Current console control schemes can be linked back to the Nintendo64 controller, which included an analog stick alongside the directional pad. This led to Sony's dual-analog controller for the PlayStation 2, which allowed players to control both the player and the camera of the game at the same time, opening up possibilities for the modern video games we see today. The function of the two analog sticks is arguably one of the most significant reasons why video games are full of first-person shooter and adventure games. Other games, as Brown notes, like real-time strategy games, "require lightning-fast access to any part of the screen" (Brown, 2016) and are just not as accessible on the home console system, like they are for a keyboard and mouse on a computer. The controller revolution we are currently in the midst of is based in advances of motion control and virtual reality, and while they are in their infancy, they have allowed for new types of games to take centre-stage. I am limiting this controller history specifically to the home console controller, but handheld game consoles and mobile gaming, with their own specific controller capabilities, have many popular games that would never find success on a home console (think of Angry Birds or Fruit Ninja on your phone or requiring the precision of a stylus on the Nintendo 3DS).

Other studies consider how elements of interactivity, immersion, and effectance are mediated through video game controls and whether they are paramount to player enjoyment of a game. Interactivity and effectance in particular are concepts specifically related to video games and how much enjoyment a player may garner from the game. Klimmt and Hartmann (2005) 
identify interactivity as, "the main feature that distinguishes computer [and video] games from most other kinds of entertainment", due to the fact that not only do "players actively process information provided by the medium [...] but they also contribute substantially to the quality and progress of the media product itself" (p. 133, 133). Klimmt et al. (2007) also acknowledge the perceived inter-relatedness of control and effectance, noting that, "one's own causal influence on the situation (effectance) is a precondition of control" (pp. 845-846). When it comes to determining a player's enjoyment of a game, however, effectance is seen as being a very "basic determinant of enjoyment" that is only affected by technical game issues that cause a player to be unable to play a game properly (Klimmt et al., 2007, p. 847). Control is a more salient element of player enjoyment, as players can find enjoyment from both being in control and having control wrested from them by a game (Klimmt et al., 2007, p. 847). For players, the challenge of not being in control produces "suspense", and having control "evokes emotional relief," both of which are "states of enjoyment" (Klimmt et al., 2007, p. 847). Understanding how different aspects of control translate to a positive play experience will be a major tool to investigate how haptic communication is utilized by the video games featured in this project.

\section{Rhetoric of Video Games}

One specific scholar provided a clear and distinct illustration of how one can find rhetoric in video games. In his The Rhetoric of Video Games, Ian Bogost analyzes both the physical construction and thematic development at work within video games. By focusing on how video games develop their thematic meaning, Bogost establishes terminology that will be significant to this project. Bogost explains that the boundaries and rules of operation of a game create the space in which a player can experience the nature of the game. This is called the possibility space, and it is further described as, "the myriad configurations the player might construct to see 
the ways the processes inscribed in the system [of the game] work" (Bogost, 2008, p. 121). The key idea Bogost is highlighting is how processes dictate gameplay, building the environment of the game and bringing meaning to the player through the gameplay mechanics themselves (Bogost, 2008, p. 121). The importance of process to game development leads Bogost to coin the term procedural rhetoric, which he understands to be the rhetorical mode in which video games establish their meaning. Procedural rhetoric, and its relationship to audio and haptic communication, will be a major area of research undertaken in this project.

One final resource that will be used by this paper takes into consideration what kind of motivating factors gamers respond to when choosing what game to play. This resource will help to distinguish different types of games from one another in the next section. One well-known taxonomy of player types is credited to Richard Bartle, who postulated that "players are of four types: Killers, Achievers, Socializers and Explorers” (Ryan, 2006, para. 8). These players subsequently fall into four quadrants of behaviour: "(a) acting (on) versus interacting (with) the game elements, and (b) focusing on other players versus the virtual world itself in one's actions" (Ryan, 2006, para. 8). This taxonomy can be applied to online games and offline games alike. In simple terms, "Killers wish to act on (i.e. kill) players; Socializers wish to interact with players; Achievers wish to act on (i.e. achieve within) the virtual world; and Explorers wish to interact with (i.e. explore and manipulate) the virtual world" (Ryan, 2006, para. 8). Through this template, one can identify players of Super Smash Bros. Ultimate as Killers, as they act on other players of the game. Breath of the Wild players, on the other hand, would primarily identify as Explorers, as the game reinforces exploration through its robust physics and chemistry engine, allowing players to both go anywhere and overcome challenges in a multitude of ways. 
Building on Bartle's player model is Nick Yee. Yee's focus is toward Massively Multiplayer Online (MMO) games, which feature hundreds, if not thousands, of players interacting with each other online via avatars (Ryan, 2006, para. 9). Through his work, Yee identified three motivating behaviours in video game players. For Yee, "Players focused on achievement seek game mastery, competition and gaining power within the game. Social players want to interact with others and develop in-game relationships. Immersion players desire to escape real life problems, engage in role-play and 'be part of the story"' (Ryan, 2006, para. 9). While Bartle's model did allow for some overlap among player behaviours - a player of Call of Duty could be both a Killer and an Achiever, as major elements of the game build on these principles - Yee's model introduces a spectrum of variability for player motivation. A player could easily be motivated by the promise of immersion in a game, while also looking to socialize with other people and achieve success within the same game. Within the physical game environments of Breath of the Wild and Super Smash Bros. Ultimate, immersion and achievement are clear motivating factors to play each game, respectively. The social element of these games - and many others for that matter - can be found in the robust online communities that spring up around specific game franchises as well as esports competitions.

I identified a number of gaps in the literature, most of which stem from the focus on video games themselves and the lack of attention given to communities surrounding video games. For example, there were many inferences to the size of online community, particularly related to the size of certain Twitch streams. Hamilton, Garretson, and Kerne (2014) talk explicitly about audience sizes on particular streams, but the authors did not evaluate the significance of community size in online environments as much as they could have (p. 1321). I think there is a gap in the literature as it relates to community size, specifically regarding how the 
community can reconcile the need for small communal groups online to foster community with the entrepreneurial sense of successful streamers, who want to monetize the largest audience possible. Another gap in the literature in my field of study pertains to accessibility. There is a lot written about the accessibility of specific video games to players, and while that is a major issue in the video game industry, there is not very much written about accessibility problems that exist within the online community of video game fandom itself.

This paper will not be spending much time analyzing the online communities that surround the games in question, but such analysis is a definite gap in video game literature. There is plenty of quantitative information to be found on online video game communities, particularly regarding Twitch, the Godfather of video game live streaming and a constant hub of video game communal activity. A qualitative approach to online video game communities falls slightly beyond the purview of this paper, but a fully fleshed out analysis of how audio and haptic elements of games becomes integrated and repurposed within the online fan communities of said games is a tantalizing follow-up to studying audio and haptic communication within games themselves. 


\section{Research Methods}

This paper is going to use a case study as the primary method of analysis. I will be studying multiple games, one that is explicitly competitive and has an active online and competitive play community, and one that is more free-flowing and is not competition-based. This case study will not only analyze the current iteration of these games, but will also take into account influential features of earlier games within the same series of games or within the same genre. The competitive games I will be looking at will be Legend of Zelda: Breath of the Wild and Super Smash Bros. Ultimate. This project will discuss elements from other games to act as support or foils for findings made about these particular games as well.

I chose Super Smash Bros. Ultimate and Legend of Zelda: Breath of the Wild as the games to study primarily because they are recent representations of two very different types of video games players play. Both games are played on the Nintendo Switch, which makes for a more compelling comparison due to the games being products of the same company and using the same system and controllers. Both games are the most recent instalments in long-running franchises, which brings historical context into the comparison as well. Most importantly, Super Smash Bros. Ultimate is a game rooted in competition, and Breath of the Wild is an introspective game about exploration, discovery, and creativity.

The research questions I have established revolve around the diversity of meaning that video games possess, and how the developers of video games go about using different techniques to communicate that messaging to their players. A video game is a complete system that puts forth the rules of engagement for the player. That system holds its own rhetoric, and developers can use audio and haptic communication to reinforce the procedural rhetoric within the game. Players are constantly being affected by both the structure of the game and the gameplay of the 
game itself. The five research questions I will be engaging with in this essay all focus on how video games can communicate with players through different modes, and how that communication works implicitly and explicitly on the player.

The case study approach was chosen for this project because of its ability to address qualitative questions such as 'how' or 'why' (Baxter \& Jack, 2008). Case studies can capture the full context that the analyzed subject is immersed in (Baxter \& Jack, 2008). I will be creating parameters for my case study through a rigorous explanation of how I will be defining playful and competitive games. This will be an exploratory case study, focused on how haptic and audio communication is being effectively used by these particular games. This will lead to a comparative analysis between the two styles of games I have laid out.

Video games vary in style and purpose enormously. The Entertainment Software Association (ESA) simplified genre categorization by listing nine supergenres, which are "fighting, role-playing, children and family entertainment, action, adventure, strategy, shooter, racing, and sports" (Collins, 2008, p. 124). All of these supergenres have differing relationships to online play: for instance, a role-playing game (RPG) utilizes online capabilities to populate their game with as many player-controlled avatars as possible in order to convey the size and scope of their game, and to enable interactivity and collaboration among players. Other supergenres, like fighting and shooter games, use online capabilities to connect real players in order for them to compete against one another, sometimes as part of a team, sometimes in a oneon-one context. While games that fall under the fighting, shooter, racing, sports, and (oftentimes) strategy genres tend to have offline modes of gameplay such as a single player campaign, the core purpose of these games is competition, be it when you invite a friend over to play or if you challenge a stranger online. The current trend of über-popular online games such as Fortnite, 
Apex Legends, Overwatch, and Call of Duty, to name a few, all consistently update their games to freshen up the competition. In the case of these four games, those major updates take the form of 'seasons', in which the games introduce new characters, maps, weapons, and game modes for players to experiment with every handful of months. The developers of these games focus on increasing updates for already published titles instead of working on new titles in the series, which exemplifies the importance of online play to these game developers.

Take the most recent release in the Call of Duty series, Modern Warfare. Call of Duty games have a history of using sequential titles to their popular games, such as Modern Warfare 2, Modern Warfare 3, and Black Ops 2. When Modern Warfare was in production, it was considered a reboot to the series. The title of the game contains no sequential numbering, even though it technically aligns with other stories within the Call of Duty universe. One could speculate that the reasoning for eliminating sequential numbering in the title is to give the game more longevity. Updates can be continuously released, and the popular esports league Call of Duty League can have a flagship game to base their competition around, without fans thinking it's weird that the flagship game they play is technically the fourth in the 'Modern Warfare' series.

As a fighting game, Super Smash Bros. Ultimate falls into this category of game. While Nintendo's design philosophy is focused on creating games that allow for a fun, easy-going multiplayer experience for everyone aged 5-95, the Super Smash Bros. franchise has grown its own devout base of competitive players (Miyamoto, 2007). Unlike the shooter games mentioned above, Super Smash Bros. Ultimate incorporates different types of sound design, mainly the use of an expansive, customizable soundtrack to go with the diegetic sounds produced through 
gameplay. On top of that, the basic controller layout also lends this game to have some interesting haptic relationships with players, which will be discussed later.

Super Smash Bros. Ultimate is the fifth instalment in the series of games from Nintendo, released for the Nintendo Switch in late 2018. Super Smash Bros. has been a popular gaming franchise for over twenty years, with the first game being released for the Nintendo 64 in 1999. A basic fighting game for all levels of player, Super Smash Bros. Ultimate, like its predecessors, is a Nintendo-specific crossover fighting game, bringing characters from many Nintendo titles into the same game to do battle with one another. Currently, Super Smash Bros. Ultimate has a cast of 74 playable characters and 103 fighting stages based on previous Nintendo games. Nintendo games tend to not be developed with online play primarily in mind (as opposed to titles like Fortnite and Call of Duty's free-to-play online experience, Call of Duty: Warzone). The same is true of Super Smash Bros. Ultimate, which does have a lengthy, monotonous single player campaign. Also, with the Nintendo Switch's controller technology, up to 16 players can play together locally. However, as I have mentioned, the online community surrounding Super Smash Bros. games is large, and as such, Super Smash Bros. Ultimate does have a large and intense network of competitive online tournaments and matches.

On the other end of the video game spectrum are games designed by developers as a more or less complete and contained game for players to enjoy. With no major updates and less of a focus on online play, these are story driven games that are designed to give a single player a specific in-game experience. From the aforementioned supergenres, it is the action, adventure, and role-playing genres that tend to be found in single player game titles. The most popular game design for these genres of games is the open-world layout, which can allow for elements of all three of these genres, including others, to exist within the same game. Popular titles of open- 
world games include any games from the Grand Theft Auto and Assassin's Creed series, Red Dead Redemption, and the Fallout and Far Cry series. This does not even scratch the surface of the amount of open-world games that are on the market. Open-world games do have precedent for being popular online games, as Grand Theft Auto $V$ remains one of the most popular online console games even though it was released in 2013. Online experiences of open-world games tend to be popular for players who want to experiment and interact with other players in the world. This type of playing has given online games of this nature the 'sandbox' title, as the freedom the player experiences within the boundaries of the game allow them to do whatever they want.

A single player game is not contingent on utilizing an open-world style to provide the player with a specific, fully contained experience. Games like The Last of Us and the Uncharted franchise - not to mention any number of horror or suspenseful games - provide the player with a linear storyline, but one that takes the player on an immersive journey. Linear gameplay is a gameplay mechanic used in the majority of video games. Linear gameplay features campaign missions that are played in a particular order. These missions can be restrictive in their content as well, keeping players from exploring the setting of the mission, guiding them in the direction they need to go. An example of linear gameplay would be the campaign mode of any Call of Duty game. The player may find themselves fighting down busy city streets, but if they try and explore the insides of the buildings on the street, they'll find all the doors to be locked. Linearity is not a bad design mechanic, but for open world games that promote exploration, having a nonlinear story campaign accentuates that exploration motif. The open-world style of games provides a more interesting palate when considering how audio and haptic communication are incorporated into the game design. Regardless of whether the game is free to explore at your own 
pace and interest (non-linear), or whether it has a strict linear structure, these games are all attempting to convey significant narrative messages and themes that support their plot, through their gameplay.

The game this project will be studying from this point of view is the latest instalment in the Legend of Zelda series, Breath of the Wild. Released in 2017 for the Nintendo Switch, Breath of the Wild is a highly inventive open-world single player game, that relies on the nature of its open-world to effectively convey the themes and messages of its story. The Legend of Zelda series premiered in 1987, when The Legend of Zelda was released for the Nintendo Entertainment System (NES). The game was a top-down, side scrolling adventure game on the 2D system. Over the next 30-odd years, Nintendo would produce 18 other Legend of Zelda titles that each varied in theme and gameplay mechanics from the previous games in the series. Some games were more ambitious and expansive than others, all with varying themes ranging from dark and gritty to pastel and peppy. For each game, the story remains relatively the same: The player controls the warrior Link, a silent avatar who is the protector of Princess Zelda. In every game, Princess Zelda gets attacked or captured by the evil Ganon/Ganondorf, and the player must guide Link through the land of Hyrule to save Zelda and the world. While the story remains the same, the elements of gameplay and design surrounding the story make each game unique. Breath of the Wild is a great game to study for the purpose of this project due to the brilliance of its open-world design. How sound and noise are employed in the game is both inventive and innovative. While the Legend of Zelda franchise has millions of fans worldwide, this version of the game is lauded by the online community as one of the best games of all time. Haptic communication in Breath of the Wild often is used to support the open-world play experience that the game emphasizes. With Breath of the Wild and Super Smash Bros. Ultimate, 
this project has two games that strongly represent two opposing poles of video game play - one that focuses on the play experience, and one the focuses on the game experience. 


\section{Findings and Discussion}

\section{Super Smash Bros. Ultimate - Haptics}

With the history of the Super Smash Bros. franchise already briefly outlined above, I want address one of the research questions of this paper. How does haptic communication methods differ based on the genre of the game? Super Smash Bros. Ultimate is an extremely popular fighting game, but the genre itself is less popular in today's video game marketplace than it was during the heyday of arcade and early home console video games. Popular fighting games from these eras, such as Mortal Kombat and Street Fighter have attempted to modernize themselves along with the current video game zeitgeist, but with diminished success. Some games, like Mortal Kombat, leaned into the violence and gore of the fighting game genre. Super Smash Bros. however, does not rely on the gimmicks of these particular fighting tropes to have success. The Smash series of games are meant for everyone, and not only in the sense that the games be kidfriendly. So, what makes Super Smash Bros. a successful fighting game?

The simplest answer is this: the games are easy to play. It is beneficial to Nintendo that they can rely on the popularity of the characters from its other popular games to help popularize Super Smash Bros. However, the games are still meant for everyone, and that means that everyone, from people who have never played a video game before to those who are professional gamers, should be able to pick up the game and play. And what do they pick up? The controller.

Video game controls have always been an essential aspect of video games themselves. As Matthew Wysocki (2013) writes in the opening of his book, Ctrl-Alt-Play: Essays on Control in

\section{Video Gaming:}

On a basic level, unlike other media, if a player does not control the game, there is no experience. You must participate to keep the story moving forward. While other media might require their audience to be mentally active, they remain physically passive. [...] A video game demands response. (p. 2) 
The player's response to video game stimuli happens almost exclusively through their controller. The controller is the main conduit in which the player facilitates control, and control is an important aspect of video gaming, as it corelates to game enjoyment. Through different iterations and designs, the controller has always allowed the player to act on this power that is specific to the video game genre.

The idea of control, particularly control through the controller, is arguably most prominent in fighting video games. Compared to the other supergenre games, except arguably for racing games, fighting games ask the player to have the most intimate haptic relationship with their controller to maximize their game experience. Other genres, like action-adventure games, role-playing games, and puzzle and strategy games require the player to adequately use their controller, but not necessarily master it. In many games within these genres - including Breath of the Wild - the controller is simply a conduit to assist in the play experience. This brings up a second research question: how is haptic communication used to support elements of gameplay in Super Smash Bros. Ultimate? To return to Bartle's player motivation model, a Breath of the Wild player is an Explorer, and while that exploration involves the controller in a very basic sense, the act of exploration happens beyond the controller. Smash players, on the other hand, are Killers, and the tool with which they kill is the controller. A player's success in a fighting game will necessarily correlate to their mastery of the controller. Sure, a player may be able to apply strategy within a fighting game to gain an edge, but a player must have a certain mastery of the controller in order to reach an elite level of gameplay. If a player were to play a game such as Breath of the Wild, they likely will encounter long stretches of gameplay in which the controller is so gently used that they barely register that they are holding it. In fighting games, however, gameplay happens in short, intense spurts, and the player is encouraged to 
engage with the controller in a similarly intense fashion, mashing buttons and jamming thumbsticks until their fingers ache. Super Smash Bros. Ultimate is primarily focused on the player's relationship with their controller.

The core controls for Super Smash Bros. Ultimate retain the simplicity of the games that precede it in the series. The player moves their avatar with the left thumbstick. This includes making their avatar jump by pushing the thumbstick up. While one hand is in charge of movement, the other handles the buttons, of which there are two for attacks, $A$ and $B$. If a player has never played the game before, all they need to know to effectively play the game are these controls. The simplicity of the core mechanics of the game make it easily accessible to newcomers, but the versatile controller mapping helps them to improve. While the mapping of the controller may not be as natural to a fighting game as the Nintendo Wii remote for Wii Boxing may have been, it is an extremely basic set up that is easy to learn quickly. A directionally mapped controller, such as the Nintendo Switch's Joycon, is usually at a slight disadvantage than a naturally mapped controller for beginners (Skalski, 2011, p. 238). In order to allow the controls to be even more accessible to the player, the entire mapping system for Ultimate can be changed by the player to fit their own style. This is especially helpful on a console such as the Nintendo Switch, since controllers can be used in a handful of different ways. The Joycons may not be as natural as the Wii remote that preceded them; Nintendo has tried their best to make controllers more naturally usable while maintaining a versatility to all different types of games the console carries.

Improving one's control over one's chosen avatar is how the player improves and succeeds at Ultimate. The player's enjoyment of the game is borne out of the tension of becoming more skilled while facing skilled opponents that test their abilities. Limperos et al. 
(2011) invokes Csikszentmihalyi's flow state (synonymous with immersion), expressing that the flow state can only occur in "optimally matched conditions of task difficulty and user-ability" (para. 11). In many other genres of video games, including Breath of the Wild, there are many other game elements that can assist in immersing the player in the gameworld. The only gameworld that Ultimate can claim to have is their single player campaign mode, World of Light, which is not a primary selling feature of the multiplayer fighting game. Ultimate is not able to rely on setting and story to help immerse the player; it has to rely on its fighting mechanics and a good skill-to-challenge balance. This process all begins with controller competence.

Discovering what each attack does, which itself depends on which of the 74 characters you are playing as, is part of the joy of playing the game. While a newcomer to the franchise may be a fan of other Nintendo games and thus have a prior relationship to some of the characters in the game, within the game itself, characters primarily express themselves through the actions prompted by the player. One of my favourite characters to play with in the game is Captain Falcon, who is the main character of the F-Zero racing series. I have never played those games before; my connection to Captain Falcon is completely rooted in Super Smash Bros. games. My attraction to playing as Captain Falcon mostly has to do with his iconic attack, the Falcon Punch. Falcon Punch is expressive and dramatic: the attack takes up a lengthy period of time (close to a second), is accompanied by Captain Falcon yelling out the name of the attack, and when the attack does happen, a flaming eagle appears around his fist. As he winds up the punch, the player feels the controller vibrate a little, mimicking the uncontrollable power of the attack. When the punch is launched, the attack is dangerous, flinging opponents across and beyond the stage with ease and rattling the controller with a satisfying vibration, letting the 
player know the attack landed. Characters in Smash rarely speak, and the campaign mode of the game does not provide backstory to any of the characters within the context of the game. Players of the game have to rely on the action of the characters to decide who they like and who they dislike. In this way, the controller is the primary method for players to connect with their avatars. To promote this kind of connection and provide more personality to the characters, the Super Smash Bros. series includes a 'taunt' system, in which if a player hits an easy combination of buttons simultaneously, their avatar will do a unique taunt directed at their opponent. These are a few of the ways in which Nintendo is able to build a connection between the player and their avatar through a haptic relationship with the controller.

While learning to play the game is easy, playing the game well can be quite difficult. For the advanced player, Nintendo built in many slightly more complex fighting mechanics into the game. If you are a beginner, you likely will not notice things like edge-guarding (when one player keeps a player from returning to the stage), footstooling (when one player jumps off the head of another player), buffering (the act of inputting moves in advance to the move being enacted), and stale move negation (the term that describes how using the same attack repeatedly in a match makes the attack exponentially less effective). These mechanics are there for more advanced players to study and practice, and they often require a much more intimate understanding of control. Mastering these techniques is essential to play Ultimate at an elite, professional level, and oftentimes requires the player to have a more intricate knowledge of how Super Smash Bros. Ultimate works on a technical, procedural level. This is not uncommon in many fighting titles.

The next research question to address is: how does haptic communication convey how the game functions to the player? For Ultimate, the question is reversed - understanding the 
function of the game can provide insight into how to better achieve control of your character in the game. The objective for ultimate control of the controller in a fighting game is a sentiment spoken to by James Ash (2012) in his article, "Technology, technicity, and emerging practices of temporal sensitivity in videogames". While the heart of Ash's essay is aimed at challenging Mihaly Csikszentmihalyi's flow theory by positing that how one experiences temporality is significantly affected by their relationship to an object (in this case, a technology), he does discuss the importance of haptic communication in this context (Ash, 2012). Ash focuses his analysis on elite players of Street Fighter IV, a fighting title developed by Capcom for the Xbox 360 and PlayStation 3 in 2009. Learning to master the controls of a fighting game is exceedingly difficult, as Ash writes, “even when competent players have learnt key aspects of their chosen character's frame data, the temporal windows in which the inputs have to be performed can still be too small for them to be performed in reaction to what other players are doing" (Ash, 2012, p. 197).

Frame data is the technical process at which all video games are run. Games consist of thousands of still frames shown rapidly every second, in order for the player to believe they are witnessing fluid motion on screen. Each game runs at its own frame rate (for Ultimate, this is 60 frames per second), and character animations for moves and attacks take a certain number of frames to complete (Falcon Punch is just below 60, meaning it takes just under a second). For fighting games, "frame data breaks each move down into three components: execution, attack, and recovery," crucial information for the advanced player, as all three components of stages of invulnerability and vulnerability to them (Ash, 2012, p. 194). Smash fights among professionals are fought on the level of frame data, as opposed to what they see on the screen in real-time. As one Street Fighter IV professional describes, “"there is no way you can react to it [an opponent's 
move], but you can anticipate it. It's anticipation over reaction"” (Ash, 2012, p. 197). This player quote is in response to anticipating an attack from an opponent. Similarly, if a player wants to land a combination of moves, they have to rely on the same kind of anticipation. When asked how he lands his hardest combination attack, the Street Fighter IV player says:

'You have to defeat your own perception of when [the inputs] should be in terms of the animation just to learn by trial and error when it will actually be. It's not a case of looking to see when his [the character Ryu's] hand and arm pulls back. You have to learn when it works and then look at the screen retroactively. You don't lead with the visual, you lead with the success of the inputs.' (Ash, 2012, p. 199)

The fight simply moves too fast for the human eye to register. In Ultimate, characters display their load-up of moves in the range of $1 / 60$ th of a second. The fight cannot be reliably fought when visual stimuli is the primary communicative tool for the player. Instead, the technical understanding of the processes involved in the game, partnered with a mastery of the controls is required. At the professional level, Super Smash Bros. Ultimate is primarily played haptically, not visually.

Many of the more advanced techniques in Ultimate are intimately haptic. They involve inputting specific directions into your controller to cut short natural character animations. One of the most common instances of this in Ultimate involves changing direction, specifically referred to in the community as a 'dash cancel'. When the player presses the left thumbstick completely in the left or right direction, their avatar will sprint in that direction. If they want to change directions, a beginner player would likely push the thumbstick in the opposite direction immediately. This makes the character perform a quick slide to stop their momentum, as they plant their lead foot and sprint in the other direction. While this is a natural occurrence that imitates the movement of a real-life person, in Ultimate the character requires multiple frames to complete the animation, leaving them vulnerable to attack. In order to avoid this, the player must quickly move the thumbstick back to the neutral position prior to their change in direction, in 
order to cancel the vulnerable animation. This is an example of how understanding frame rate and other technical aspects of the game, partnered with how those aspects relate to the controller, is crucial to improving one's ability at the game. Elite players of Smash constantly need to input multiple commands on their controller within quick succession of each other. The commands themselves can involve complex and intricate motor skills, such as circling the thumbsticks in different patterns simultaneously to cancel out of certain animations in order to land attacks quicker.

There is only so much for the player to control within a Smash Bros. fight. The setting is only a backdrop to the competition at hand. The only thing a player has any kind of effect on is the control of their avatar and how that avatar interacts with the opponent's avatar. With so little available to control in the game, having the requisite skill to control your avatar better than your opponent is paramount to game enjoyment. As Klimmt (2007) notes, "players enjoy watching the results of the actions they perform" (p. 847). Without a setting or a story to assist in bringing an immersive experience to the player, Super Smash Bros. Ultimate has to rely on the delicate balance of providing players with challenging obstacles that match their skill with a controller. With a minimum of other gameplay elements and game design techniques to help in capturing this immersive quality, Ultimate is a game that lives and dies by the controller. The Smash series, however, also provides an interesting substitute to the lack of setting and story in the game by providing fans of the game with a real-life competitive esports narrative and online community setting.

The popularity of tutorial videos speaks to the importance players put on the controls of Super Smash Bros. Ultimate. Not only are players keen to learn more advanced skills, but advanced players are providing other players with that guidance. This kind of relationship 
follows the pattern found in video game online communities in the article "Narratives, identity and community in esports," by Xue, Newman, and Du (2019). This article outlines the desire for established members in the video game community to guide and assist newer members of the community, partly out of their own desire to be considered an expert (Xue et al., 2019). Not only that, but the authors found that established members of the community tended to be entrepreneurial and looked to these newer members as potential consumers (Xue et al., 2019). Advanced players posting tutorials on how to better play the game, including offering paid online tutorials to potential players, is not uncommon to find on YouTube or Reddit.

The relationship between players and other players within the Super Smash Bros. online community, and between the player and the game itself, is ultimately moderated by the controller. There is a sense of lore behind the characters that esports athletes play particularly well, with character popularity often being swayed by who plays them publicly. This lore can provide some narrative to the gameplay of Ultimate for those who care enough to know about it, but in general, the game is designed for anyone to pick up and play. The principle desire of Super Smash Bros. Ultimate is to be an accessible, fun, cooperative, and competitive experience. By supplying players with a high number of characters and stages to play, players are prompted to try and master multiple characters for the variety of locations a fight might take place. The game experience is maximized when a player has a haptic relationship with their controller that is precise and smooth. The goal of the game is to beat your opponent, be it a computer or a friend, and the best way to achieve that goal is by perfecting the controls of the game. In order to perfect those controls, a player can turn to the online community, as much of the popular content created within the community is directly tied to the haptic relationship the player develops with their controller throughout their gameplay. While the game itself does include many tips and 
information on the abilities of each character, online tutorials provide more in-depth analysis of this information, and often show visual examples of what the player is trying to learn. The player-controller relationship is a fundamental component of video games as a rhetorical device. As Matthew Wysocki (2013) writes, “[...] control has a larger implication of power inherent in it. When we bring control into the picture, we are not just interacting with an avatar; we are commanding it, directing it. We are attempting to enforce our will into the game world, not just acting within it" (p. 2). The controller is the player's primary communicative tool within the video game, and in fighting games, that relationship is seen most starkly.

\section{Rhetoric of Control}

In his essay The Rhetoric of Video Games, Ian Bogost (2008) discusses the specific kind of rhetoric employed by video game designers to give their game more substantial meaning. Bogost coins the term procedural rhetoric to describe this development of meaning. Bogost (2008) emphasizes procedure because it describes:

[...] the practice of authoring arguments through processes. Following the classical model, procedural rhetoric entails persuasion-to change opinion or action. Following the contemporary model, procedural rhetoric entails expression-to convey ideas effectively. Procedural rhetoric is a subdomain of procedural authorship; its arguments are made not through the construction of words or images, but through the authorship of rules of behavior, the construction of dynamic models. In computation, those rules are authored in code, through the practice of programming. (p. 125)

This understanding of procedural rhetoric can easily be applied to video games, where designers are responsible for developing symbolic systems that create the rules within which the player is free to play (Bogost, 2008, p. 121). It is the rules created by these processes that the player is constantly operating alongside or against. Bogost (2008) explains that, "video games represent processes in the material world - war, urban planning, sports, and so forth - and create new possibility space for exploring those topics. [...] We encounter the meaning of games by exploring their possibility spaces. And we explore their possibility spaces through play” (p. 121). 
While some games take this responsibility of design more seriously, procedural rhetoric is still used by games to "create an entertainment experience, a fantastic situation that transports the player to another world" (Bogost, 2008, p. 123). Whether done deliberately or inadvertently, providing players with a structure in which they can play is an act of procedural rhetoric (Bogost, 2008, p. 123). The game developers express themselves through the game structure, and the players get to grapple with that expression through their gameplay.

It would be difficult to argue that Super Smash Bros. Ultimate contains any kind of meaningful procedural rhetoric (this will be investigated more fully when we discuss Breath of the Wild). The game's processes promote highly entertaining gameplay. However, with the importance that the game and the online community surrounding the game places on the player's haptic abilities, one could argue that the game's most significant rhetoric speaks through the controller. Video games commonly grapple with themes such as control and agency, as the medium of video games necessarily relies on action from the player. Some games use these themes to question aspects of morality; a game like Call of Duty: Modern Warfare 2 famously included a campaign mission in which the player is directly implicated in a massacre of innocent civilians at an airport. While the moral issue that arises from a mission that can seem powerful on the surface, they ring hollow because of the lack of agency the player actually has in the moment. If the player wants to continue the game, they have to participate in that mission. There is no alternate gameplay path that allows them to make their own choice and live with the consequence. They could stop playing the game entirely, but that is a decision of agency that takes place outside of the parameters of the game as purposefully designed by the game developers. It is ideal for a game to explore ideas of control and agency through the unique medium that video games provide, namely, gameplay. 
Matthew Wysocki's book Ctrl-Alt-Play: Essays on Control in Video Gaming, grapples with the question of gameplay and control. In the book's first essay, penned by Gerald Voorhees (2013), gameplay is "more than simply an intersection between an agentic player and the structure of the game; gameplay is the agonistic struggle - playful but consequential - out of which meaningful human action emerges" (p. 16). Even the term 'gameplay' "displaces both player and game with an emphasis on the interfacing of the two," an interfacing that physically takes place through the controller. (Voorhees, 2013, p. 16). There are some games that are successful in applying their procedural rhetoric directly through the controller. The Last Guardian is the third game designed by famed auteur game designer Fumito Ueda, who has a reputation for placing procedural rhetoric in his games that "evoke empathy in the game through play itself, rather than through dialogue or animation" (Suellentrop, 2011, para. 3). The Last Guardian has the player controlling a young boy, who befriends a gigantic, half-bird half-beast mythical creature, named Trico. Throughout the game, the player explores the ruins of an unknown city with the help of Trico, but the player never gets to control Trico. The player must communicate with the character through gameplay mechanics, namely petting and comforting Trico with controls on the controller. However, how the player interacts with the controller is often counter-intuitive. Video game critics Ben Lindbergh and Jason Concepcion (2016) discuss the controls in their joint review of The Last Guardian for The Ringer, a pop culture and technology publication. Concepcion notes that there is an "imprecision to the way the left [thumb]stick moves the boy through the world," and, to a seasoned video game player, the controls seem "illogical" and "unnatural" (Concepcion \& Lindbergh, 2016, para. 13). By using a deliberately unnatural controller layout for the player, Ueda is causing the boy in the game to behave more like a real child would behave in the game environment, as opposed to the type of 
smooth and seamless behaviour of countless video game characters players are used to. As Concepcion determines, "the deeper reason for crafting the game this way is the clunky controls enhance the feeling that you are playing as a child" (Concepcion \& Lindbergh, 2016, para. 16).

Not only does the controller affect the player's relationship with their young avatar, but the player's attempts to indirectly control Trico through the boy is equally foreign to video games, but similar to life (Concepcion \& Lindbergh, 2016, para. 17). Lindbergh noted during his gameplay that when the player calls Trico, the AI of the animal is not fully guaranteed to respond to the command. Trico probably responded immediately and exactly to his command about a third of the time (Concepcion \& Lindbergh, 2016, para. 22). By rendering the controller to be randomly ineffective, The Last Guardian causes the player to grapple with the idea that the processes of the game are not always perfect, and that they cannot fully rely on their own abilities to complete the game. But, as Lindbergh highlights, "if Trico were a seamless extension of the player's will, he might not feel like such a full-fledged companion. [...] I don't think I've ever become so attached to a non-humanoid [non-playable character]" (Concepcion \& Lindbergh, 2016, para. 24). While Ueda's first two games grappled with themes of companionship and intimacy, and selfishness and regret through their gameplay respectively, The Last Guardian employs the player's own intimate relationship with the controller in order to further establish its own rhetoric of "developing a relationship with nature and the power of kindness" (Concepcion \& Lindbergh, 2016, para. 3).

Chris Suellentrop (2011) writes in his New Yorker article, “The Video-Game Art of Fumito Ueda", that "as video games have become more and more popular, the medium's defenders have developed a misguided tendency to point to the ways that games are useful, practical, functional" (para. 14) This is indeed misguided, as Ueda and many other game 
developers can prove with the content of their games. Procedural rhetoric gives value to the game experience, as Bogost (2008) writes:

[...] video games are not just stages that facilitate cultural, social, or political practices; they are also media where cultural values themselves can be represented-for critique, satire, education, or commentary. When understood in this way, we can learn to read games as deliberate expressions of particular perspectives. In other words, video games make claims about the world, which players can understand, evaluate, and deliberate. (p. 119)

Video games can indeed be practical as much as they can be engaged with important cultural and social questions. And, of course, games can be fun. Super Smash Bros. Ultimate does not have a procedural rhetoric with the same kind of weight as The Last Guardian. They are different games serving different purposes. However, one can find some meaning in the way Super Smash Bros. Ultimate prioritizes player control through the player's capabilities with the controller. As a game designed for fun competition, the goal of the player is to get as good as they can. While some of this improvement falls to strategizing within the game - such as deciding which character to use in certain matchups at certain stages - the majority of improvement is related to controller mastery.

\section{Breath of the Wild - Haptics}

The player's relationship to the controls of The Legend of Zelda: Breath of the Wild is much different than their relationship to the controls of Super Smash Bros. Ultimate. Mastering the controls of Ultimate is the most significant way a player can improve their skills in order to beat both the single player campaign and their opponents in multiplayer. In Breath of the Wild, mastering the controls is not an essential aspect of improving one's ability at the game, and does not hinder a player from completing the game. While the controller is the primary method through which Ultimate communicates with the player, the controller's role in Breath of the Wild is to provide an easy, seamless way for the player to explore the world of the game. In order to consider the haptic communication methods used based on the genre and style of Breath of the 
Wild, this section will begin by describing the history of the development of controls within the Zelda franchise, culminating in the intricate controller layout for Breath of the Wild on the versatile Nintendo Switch. Next, this section will illustrate how aspects of control, such as effectance and immersion, are supported by the haptics of the game. And lastly, I will discuss how the idea of control is implicated within the gameplay of Breath of the Wild, moving the idea of control from a purely haptic understanding to one that is baked into the themes of the game.

The 18 games Legend of Zelda has produced over 30 years has spanned many different video game consoles, and thus, includes many different iterations of controller layouts. Zelda games are narrative and exploration-heavy, which is a stark contrast to the elements of gameplay employed in the Smash series. With regards to Bartle's player model, players of Zelda tend to fall in the Explorer and Achiever category, the dimension of the model most associated with the game environment (Ryan, 2006, para. 8). Yee's player model is even more descriptive of what a Zelda player is looking for, finding them to be both achievement and immersion-oriented (Ryan, 2006, para. 9). Typical Zelda gameplay will see the player-controlled protagonist, named Link, left to explore an overworld, which is peppered with puzzle-filled dungeons, in which the player finds a new item that they can use to progress forward in the game past previously established obstacles. The mixture of puzzle solving, combat, freedom of exploration, and story are the major gameplay elements that make the franchise so popular. These elements were initially established in the titular Legend of Zelda, released on the Super Nintendo Entertainment System (SNES) in Japan in 1986 and North America in 1987. The game, generally hailed as one of the most influential video games of all time, contained what would become core staples of the franchise and modern video gaming overall, such as non-linear gameplay, save files, and the freedom for the player to complete the game differently every time they play. The game is also 
considered to be the inspiration for the modern role-playing game genre that is overwhelming in today's video game market.

Breath of the Wild, while the $18_{\text {th }}$ game of the series, was considered by the developers to be a return to the core gameplay elements initially introduced back in the 1980s. Legend of Zelda sequels tended to blend the original gameplay of the first game with slightly different variations of story and game design. The freedom of exploration allotted to players by nonlinear gameplay became neutered in many of the sequels, as the player could only move forward to different areas of the overworld when they accessed specific items.

Breath of the Wild did away with the linearity of some of the recent Zelda titles, introducing nonlinearity back into the gameplay in arguably the most successful way in modern video gaming. Linear and non-linear gameplay can have differing affects when it comes to a player's immersion and effectance motivation. A good example of how linear gameplay can be an easy-to-use tool for immersion is in the game series Gears of War. Gears of War is a postapocalyptic first-person shooter with a strong, propulsive narrative. Each mission in the game is labeled as a chapter, and much like a work of literature, the game has a momentum that builds throughout the chapters. Like chapters in a book, the game chapters in Gears of War frequently have cliffhangers, set pieces, revelations, and tragedy. Many chapters end in cinematic cut scenes, accentuating the emotional beat of the story and pulling the player deeper into the story of the game. Linear gameplay does not necessarily allow players the freedom to explore, but the structured nature of the gameplay can be specifically designed to affect players much like a book or movie. This can be an effective narrative device for many video games, but it can lead many other gameplay elements, such as effectance and autonomy, to ring hollow. 
The golden goose in the gaming industry is that of "presence, or providing the player with a sense of non-mediated 'immersion' in a game environment” (Ryan et al., 2006, para. 98). Linear gameplay provides a mediated immersion borrowed from other art forms, like literature and cinema. To immerse a player in a game containing non-linear gameplay alongside a narrative that meshes with the autonomy of nonlinear gameplay is a feat that is achieved by Breath of the Wild. The tools for immersion are taken out of the hands of the game designers and placed in the hands of the player, and for designers, that can be a leap of faith that is difficult to overcome. Control design is an essential part of the immersion equation, as "games must allow players to focus their energy on game play rather than game mechanics (i.e. intuitive controls)" (Ryan et al., 2006, para. 98). Ryan et al. (2006) argued that immersion can also be associated with "autonomy to pursue in-game goals and interests and the competence to carry out effective actions" (para. 98). In their study, when players felt that sense of autonomy and competence, the greater the feeling of presence. Autonomy in a game is manifested in the design, whereas competence is related to the marriage of decision making and control scheme, a mixture of the game design and the player.

Some highly touted design aspects of Breath of the Wild are its physics and chemistry engine. Physics engines are common parts of game design, as the engine defines the terms of how physical objects in the game environment interact. The chemistry engine is specific to Breath of the Wild, and its role in the game is to promote player creativity and autonomy. The game imitates real-world chemistry, which can lead to random changes in gameplay. If a player gets caught in a thunderstorm, they must remove all of the metal armor or weaponry they may have equipped, or they will attract a lightning bolt. If a player shoots an arrow through a bonfire, that arrow will catch fire, land in the grass, set the grass ablaze, and the subsequent fire will 
create an updraft of air the player can use to paraglide. Understanding how the chemistry and physics engines work can provide players with a strong feeling of effectance, as they can manipulate elements of the gameworld around them in realistic ways. The autonomy given to players through this effectance motivation can be a powerful immersive tool. The chemistry and physics engine can also create a realistic sense of helplessness at times as well, as the player may be at the mercy of the weather at times in the game. While this does not create a feeling of autonomy, it does foster in the player the idea that the game is not a routinized system, but one that adapts and changes in real time.

How do video game designers use haptic communication to support elements of gameplay in their game? With the player being handed all of the items they need to play the game right at the start, the controller immediately becomes a complex array of buttons and triggers for the players to become accustomed to. With so much area to explore, Breath of the Wild does employ some very handy controller shortcuts to keep the player from spending too much time in the game menu. A common problem in modern video games is the amount of time players are forced to spend in game menus organizing inventory and equipment, not to mention constantly orienting themselves on the game map. The frequent suspension of gameplay to complete these tedious organizational tasks can be a momentum-killer, but gamers have grown used to the trope. Breath of the Wild provides some controller layout solutions to this problem in order to keep the player immersed in the open world exploration the game encourages.

Breath of the Wild has multiple shortcuts on the controller that allow players to access portions of the game menu without exiting out of the game. For the game designers, shortcuts are a helpful tool to avoid frequently disrupting gameplay and maintaining the flow state of the player. The controller should never be a barricade to the game for the player (Ryan, 2006). This 
is a crucially important device when partnered with a new gameplay element introduced in the game: weapon durability. Every weapon in the game (excluding a single sword), including bows and shields, shatter after a certain amount of use. As such, the player will routinely have to access their inventory to replace broken items, often in the heat of a combat encounter. By hitting one of the directional pad buttons, the player pulls up their inventory menu, which takes up a small amount of screen space. When the menu is pulled up, the gameplay slows to an infinitesimal crawl, giving the player ample opportunity to change their loadout without heavily disrupting the momentum of the game. This is one significant way in which the innovative controller layout for Breath of the Wild supports the gameplay desired by the game developers. The game environment is meant to be experienced and explored as smoothly and seamlessly as possible. Avoiding game menus is a great way to keep the player immersed in the primary play experience.

With regards to the map, Breath of the Wild does some simple, effective things to make navigation and orientation easy for the player. The map of Hyrule is available to the player through a single button click and has a small number of icons that one can place on the welldetailed landscape to highlight specific places of interest and importance. The most significant icon available to the player are five colourful beacons the players can apply both in the overworld and on the map screen to signify notable destinations. By letting the player mark destinations in the overworld, the game developers can keep the player from stopping play to check the map screen to verify and mark destinations. The player is encouraged to explore the overworld without spending much time in the map screen. By capping the colourful beacons at five, the game developers ensure that players don't overwhelm themselves with waypoints and things to do, and actually achieve objectives and reach destinations before moving on to another 
one. By including multiple shortcuts to sidestep the player having to spend time in their game menu screen, the Breath of the Wild game developers understand that the controller layout can be used in order to better facilitate the experience they want the player to have.

Adapting these controls to support gameplay is a simple, but effective design choice. The Breath of the Wild controller layout also takes advantage of the innovative new technology provided by the Nintendo Switch console. On every Nintendo Switch controller, there is a small motion control system at the end of the device. Breath of the Wild allows players to use these motion control systems in their gameplay. In theory, being able to aim your bow and your items by pointing your controller at the screen is exciting and engaging, but in reality, the concept is a bit hit or miss. Players unwilling to utilize this new technology can rely on the usual operation of the right thumbstick to help direct their aim. However, the motion control technology remains in effect, leading to frequent moments where aiming is difficult to control with the thumbstick and the motion control system fighting against one another as the player tries to aim at the desired target. In addition to this tension, occasional puzzles in the game require the player to use motion control to manipulate platforms in very intricate ways. This can be a frustrating experience with a less-than-perfect system, and there are no alternative methods to control the platforms, though the puzzles themselves are ultimately optional.

Breath of the Wild has four main puzzle areas for the player to solve, on top of 120 other smaller puzzles scattered throughout the overworld. The four puzzles provide a shift in how the game functions outside of the puzzles - a shift that is conveyed through haptic communication. As with everything in the game, completing the puzzles is optional for the player, though it does make the game's final encounter slightly easier. The four main puzzles are contained within four gigantic mechanical beasts that stand out in the expansive landscape. The primary puzzle 
mechanic the player must master is manipulating the beasts themselves from the inside, solving small puzzles inside the beast by causing parts of the animal to move. Through this conceit, Breath of the Wild forces the player to grapple with the theme of control. The majority of the game has the player experiencing themes of loss and memory as they traverse a world mourning the loss of their civilization. When the player encounters these beasts, the animals are unruly and dangerous. The player must go through an action to calm the beast down before entering the machine. Once inside, the player must physically control the movement of the animal to help rid the beast of the evil infestation residing within. The player, ironically, controls the beast through the game menu. By making the player suspend their gameplay in order to precisely move the animal, Breath of the Wild uses this procedure to slow the player down and let them think of how they should maneuver through the puzzle.

The four beast dungeons are the only instance in Breath of the Wild where the game developers bind the player to a strict formula of gameplay. As Voorhees (2013) emphasizes in his essay, "game designers do not directly design play. They only design the structures and contexts in which play takes place, indirectly shaping the actions of players" (p. 18). Breath of the Wild accomplishes this mandate throughout its gameplay. The overworld is an immaculate sandbox in which the player can carve out their own personal play experience. The player's experience within the possibility space is "bounded by but not bound to game rules" (Voorhees, 2013, p. 18). So, when the player does come across one of the beasts, the sudden imposition of stricter rules of operation communicates a clear thematic shift - the player, accustomed to a freedom and ease of exploration in the wild of the game, is within a great beast of nature that is constructed from advanced technology. The player is forced by the game to act deliberately and 
with control when inside the beast, expressing an idea of the variability of control to the player through the procedure of the game.

Unlike Super Smash Bros. Ultimate, where the player is prompted to play the game in a similar fashion to other players, with the only difference being character preference and controller prowess, Breath of the Wild supplies the player with all the tools they need to play the game from the outset, and lets them play the game however they please. While games like Minecraft and the Hitman series are also constructed on this free, sandbox style of play, Breath of the Wild is still successful in telling a compelling narrative in a game that does not force the player to ever encounter that narrative if they choose not to. In Super Smash Bros. Ultimate, the player's haptic relationship to their controller is as intimate and primary a communicative method as a game can have; in Breath of the Wild the player's haptic relationship to the controller plays a much more supportive role to the primary elements of the game, namely, it's open world and non-linear gameplay that focuses on exploration.

Breath of the Wild control tutorials are much less popular than Super Smash Bros. Ultimate control tutorials. One could attribute this to the reliance Ultimate has on the player's ability to use their controller well, whereas Breath of the Wild has a rich narrative and soundtrack that tends to get more online attention. While Ultimate online content is closely tied to gameplay, Breath of the Wild content is more connected to lore and personal player experience. Where the idea of lore in Smash is more likely to be based in the real-life competitive scene of Smash tournaments, Breath of the Wild's online game presence is minimal, as it is designed to be single player game and experience. The players of Breath of the Wild experience the game on their own. If they want to connect with others about their experience and to hypothesize about the mystery of the game, they turn to online communities to fulfill that need. Legend of Zelda creator 
Shigeru Miyamoto encouraged this very interaction when developing the first Legend of Zelda game, saying "I wanted [players] to talk with other Zelda players and exchange information, ask each other questions, find out where to go next. That's what happened - this communication was not a competition but it was a real-life collaboration" (Brown, 2017). With Breath of the Wild being a return to that game's roots, it has a narrative that is shrouded in mystery. Not only that, but the overworld itself is a mystery oftentimes to the player, containing ruins and locations reminiscent of Zelda games past. All of this content is fodder for the online community, with much of the content surrounding Breath of the Wild hypothesizing what the answers or truths to these mysteries are.

The Miyamoto quote touches on what the foundations of the online communities are for Smash and Zelda - competition and collaboration, respectively. While the communication among the community surrounding Smash is not competitive in nature, the content is focused on the nature of competition - on becoming better, on watching and commenting on competitions, etc. The difference in online community stems from the experiences of play established by both games, and by their rhetorical content and messaging within the context of the game. As has been discussed above, the haptic relationship the player has with the controller is used by game developers to further establish or support the gameplay of each game, which in turn is one of the central processes in which the game establishes a meaningful rhetoric. Both games have succeeded in this capacity to varying degrees; moving forward, this paper will examine how audio communication can also play a foundational role in establishing and supporting gameplay structures and procedural rhetoric in modern video gaming.

\section{Breath of the Wild - Audio}


If you consider yourself one of the millions of people worldwide that are fans of the Legend of Zelda series, you likely have already experienced a complete history of how music and sound design has evolved in video gaming. From the first Legend of Zelda through to the Legend of Zelda: Breath of the Wild, this series has showcased the variety of ways in which sound and music can be utilized, both technically and artfully. Of course, the development of new technology has a lot to do with the evolution of music and sound design in video games - much of this history can be charted through Zelda games as well, from the limitations of the 8-bit era of the NES to the potential of the live orchestral recordings used for Breath of the Wild on the Nintendo Switch.

While the casual Legend of Zelda fan may not be privy to the technological advancements and compositional achievements that are essential to the success of the music and sound design of the games, they will likely tell you about how much they love Zelda music. Scroll through the comment section on any YouTube compilation of Zelda songs and you will come across comments like this from Patrick's Music: "I have a lot to say about zelda music: 1) It's amazing. Every game I continue to go “woah that's amazing." 2) I love Zelda's music team. Somehow they continue to write beautiful tracks and record them with amazing grace and beauty. 3) The way each track is so unique and calm takes my breathe every time ..." (Vapidtomcat, 2016). Fans will argue amongst themselves, or with whoever will listen, about which Zelda game has the best soundtrack. Critics weigh in, such as Matt Peckham from Time, who wrote of Breath of the Wild's soundtrack, “composer Manaka Kataoka's restrained, often poignant musical passages are a tour de force of complementary minimalism” (Peckham, 2017, para. 10). Fans will buy tickets and watch an orchestra play their favourite songs. And, perhaps 
most significantly, they will tell you how important the music is to their experience of playing the game.

Music and play share many commonalities. As William Cheng (2014) writes in his book on the topic, "both gameplay and musical play involve the apprehension, interpretation, and manipulation of signs and materials within technical constraints and customs" (p. 4). Johan Huizinga (1971), famous for his considerations of play, even describes the act of play through musical language, saying, "[play] is invested with the noblest qualities we are capable of perceiving in things: rhythm and harmony" (p. 22). Huizinga also describes play as being essentially free and, secondly, “that play is not 'ordinary' or 'real' life. It is rather a stepping out of 'real' life into a temporary sphere of activity with a disposition all of its own" (Huizinga, 1971, p. 19). Like other forms of media, such as opera, film, theatre, and literature, video games immerse their player in a very realistic sphere of imagination. Unlike those other media forms, video game play can bring the player into a more dynamic and active relationship to audio. Cheng (2014) notes that, "much of the appeal of video games comes from their ability to translate our physical input into extravagant sounds and onscreen animations" (Cheng, pp. 3-4). Video games, like music, involve elements of "performance, collaboration, and competition," (Cheng, 2014, p. 4) while also "[accommodating] solitary, contemplative engagement: both are said to have therapeutic, rehabilitative, and analgesic effects; both have potential to facilitate trance, flow, and immersion; and both possess transportive powers, ushering us into otherworldly spaces and alternative states of mind" (Cheng, 2014, p. 4). Video games rely on the influential power of music and sound to add both feeling and function to the game experience. 
Sound design is a major element of game design. Sound and music are often employed to support everything from technical gameplay to the rhetorical significance of the game. Nacke and Grimshaw (2011) summarize different uses for audio, writing:

Sound and music are generally known to enhance the immersion in a gaming situation (Grimshaw, 2008a). [...] and it is generally known to trigger the mesolimbic reward system in the human brain (Menon \& Levitin, 2005), allowing for music to function as a reward mechanism in game design and possibly allowing for reinforcement learning (Quilitch \& Risley, 1973). (p. 265)

Playing video games is an emotional experience, whether the games cause the player to feel fear, joy, guilt, relief, or any other feeling on the emotional spectrum.

The communicative uses of audio in video games has developed into a nuanced element of design that is worth a game developer's thought and effort. However, the potential for what sound design can do has grown along with the technological advances in game sound. Much like the video game controller, game sound has its primitive roots in arcade games, though arcade games in turn took inspiration from early slot machines when it came to sound (Collins, 2008, p. 7). Collins (2008) notes two significant purposes for sound design in arcade games, the first one being "an attract function, which would call players to the machines when nobody was using them" (p. 9). Once the player was playing the game, Collins explains that, "sound was a key factor in generating the feeling of success, as sound effects were often used for wins or near wins, to create the illusion of winning" (Collins, 2008, p. 8). In this way, sound was purely a marketing tool to bring in players and to keep them wanting to play. The technology of arcade games limited the potential of sound design severely. The games themselves took up most of the space on the arcade game's processing system, which usually meant that non-diegetic (sound external to gameplay, such as a soundtrack), non-adaptive (sound that is not dependent on elements of gameplay) sounds were impossible to create due to the lack of flexibility and space on the machine. 
As video game systems improved and moved into the home, consoles began to develop exclusive processors for the music and sound of their games. The increase in sound flexibility allowed game developers to actually focus on unique compositions for their own games, even if they remained limited in the kinds of sounds available to them. One significant development was the potential for looping music and sounds, which referred to the possibility for a composition of sound to be looped, creating a continuous track of sound or music. Looping was a major factor in the rise of diegetic music taking place during gameplay, while still remaining non-adaptive. Collins (2008) describes the methods in which looping were standardly used, writing, "looping was also dependent on the game state - whether a player was in battle, on an easy level, or entering a new area, for instance" (Collins, p. 28). Overworld themes, like the Legend of Zelda's overworld theme, would be longer compositions than boss fight compositions. Due to their short duration, boss fight compositions could afford to be a bit more complex, as they did not have to worry about listener fatigue or space. Even with the significant shortcomings of early audio technology, Collins (2008) does enforce that developers and composers still constantly made audio decisions based on aesthetics, writing:

The persistent practice of looping is particularly illustrative of the tensions between technology and aesthetics. [...] Looping was not the only choice, however, and other approaches such as random sequencing were also seen. Looping, then, appears to be as much an aesthetic choice as a pre-determined factor led by technology. (p. 34)

For Collins, it is quite clear that video game audio was considered by developers to be an important artistic part of the game experience, and warranted the kind of attention given to decisions regarding gameplay development.

In many games, sound design is an essential ingredient to make a video game work. Sound design can be divided up into a handful of categories depending on what the sound is being used for. Collins outlines two of the significant types of audio, interactive and adaptive. 
Interactive audio, she writes, "refers to those sound events that react to the player's direct input," (Collins, 2008, p. 4) while adaptive audio, "on the other hand, is sound that reacts to the game states, responding to various in-game parameters such as time-ins, time-outs, player health, enemy health, and so on" (Collins, 2008, p. 4). An example of interactive audio is the sound Mario makes when the player presses a button to make him jump, while an example of adaptive audio is the increasing speed of the game music when Mario's time to complete the level gets diminished. Two other factors to consider when thinking about video game sound are diegetic and extradiegetic sound, the former relating to the player's "conscious interaction with the interface," (Collins, 2008, p. 3) and the latter referring to "a corporeal response to the gaming environment and experience" (Collins, 2008, p. 3).

Video games aim to be immersive experiences for their players. Unlike other mediums, however, video games interact with players, leading them to be complicit in video game action. The player is not a passive receiver of a sound signal, but often "also partly the transmitter of that signal, playing an active role in the triggering and timing of these audio events" (Collins, 2008, p. 3). While the overall experience of playing a game aims to be immersive, "the body cannot be removed from the experience of video game play" (Collins, 2008, p. 3), giving the player a relationship to sound that in certain instances goes beyond that of film or television.

The most obvious example of the interactive relationship between sound and player are in popular games that give players a choice in music. Games like Grand Theft Auto V and Fallout 3 are RPG games set in open worlds in which the player has access to an in-game radio. In GTA $V$, the player is given a large selection of radio stations to listen to when they drive around in their car. The radio stations have a limited amount of songs per station, but this kind of choice allows the player to literally set the tone for their personal game experience. Additionally, whenever a 
player steals a car in the game, the car's radio will be playing a station that corresponds to the character's role in the game. Unfortunately, this kind of specialization can enforce racialized stereotypes: for example, if the player were to hijack a cab, the radio might be tuned to a foreign language station. As Miller (2007) notes, "the player-controlled radio stations not only increase the verisimilitude and immersive qualities of each gameworld, but also encourage players to associate particular music with particular characters and places" (p. 404). This particular example illustrates how audio communication in video games can cut both ways: sound can be expressive and immersive, but it can also be stereotyped.

William Cheng (2014) discusses the significance of the radio as the primary musical outlet for the player in Fallout 3. Fallout 3 is an open world RPG set in a post-apocalyptic American landscape. Similar in function to Grand Theft Auto $V$, the player has access to a radio, which only plays a handful of different stations. Unlike Grand Theft Auto V, Fallout 3 uses the radio feature for world building, accentuating the plot, and determining the tone and feel of the game. The two major radio stations in the game are operated by competing factions the player frequently encounters throughout the game. If the player is tuned to those stations, they will hear lots of in-game news in the form of propaganda coming from both factions. If the player chooses to tune into one of the other two channels available for the majority of the game, they will hear a very short list of pre-World War II songs on one, and a short list of classical songs on the violin on the other. The sparse selection of stations and the small variety of songs is the result of specific decision making by the game developers. Cheng (2014) highlights the focal point of sound in Fallout 3, noting that "music and voices from the radio bestow a sense of imagined community by promising that somewhere, someone else is listening to the same thing" (Cheng, p. 22). While using radio as a medium for sound in the game may provide the player with this 
hopeful feeling in a barren, desolate game, when the player encounters NPCs, they are often struck by the opposite feeling. As Cheng (2014) writes:

In this wasteland, the tedious recycling of music from the past stems, players might imagine, from the unwillingness or inability of traumatized survivors to create new tunes following global atrocity. The shock of nuclear war appears to have drained all artistic impulse from the world, with day-to-day survival overtaking creative activity in the hierarchy of basic needs. Any unease a player might harbor toward the radio's mechanical music stands to be compounded by how nobody else in the gameworld seems to be listening to it in the first place. (p. 23)

The way audio is delivered to the player in Fallout 3 is supporting the rhetoric of loss and memory running through the post-apocalyptic game. While the player travels across the sparse, dangerous landscape, the only accompaniment they have are the repetitive songs of a bygone era. Post-apocalyptic video games frequently pop up on gamers' radars. Games like Fallout 3 usually bring about familiar thematic questions. Cheng (2014) illustrates the nature of apocalyptic games, saying:

Earthshaking circumstances necessarily do violence to our fundamental beliefs, priorities, and values. Simulations of end times, as such, furnish opportunities to proceed from an epistemological ground zero. How can crises of music in postapocalyptic scenarios deepen our understandings of sound and play? (p. 24)

While Fallout 3 addresses this question through the ahistorical smatterings of a radio memorializing the only past available, Breath of the Wild addresses thematic questions of a postapocalyptic world through a more composed auditory lens.

When the game first hit shelves, Breath of the Wild's soft, mournful score was extremely divisive. The scope of the game was bombastic, and the trailer for the game was action-packed. Fans expected a heroic score to accompany the hero of their story, much like The Legend of Zelda score had been back in the mid-1980s. Music has always been a foundational element of the Zelda series, with multiple titles, including the critically acclaimed Ocarina of Time and Link's Awakening, in which playing a musical instrument is a major part of the story. The fan 
community had high expectations built on perhaps the most famous game soundtrack of all time, only to find that soundtrack either missing or completely re-interpreted in Breath of the Wild. In a game so focused on memory, loss, and guilt, Breath of the Wild was able to play with these themes within its own relationship to the Zelda music canon, with Breath of the Wild sounds often being allusions to or deconstructions of classic Zelda songs.

The Breath of the Wild soundtrack has a lot of eccentricities. One of the most notable elements of the score is how frequently it is completely absent from the game. The overworld of Hyrule is massive; with the player potentially spending hundreds of hours exploring the landscape, Nintendo decided to have the soundtrack randomly cut into the quiet of the game. The reason for a lack of constant music could be to avoid players becoming tired of hearing the same music repeatedly. When the music is quiet, the player is not left in a silent world. The sound design on Breath of the Wild is incredibly well-detailed, with the sounds of footsteps, wind, and shifting equipment on Link's body creating a soundtrack of their own. As highlighted in his video on Breath of the Wild sound design, composer and 3D artist Scruffy explains that in order to more accurately capture Link's stealth abilities, the sound designers actually recorded themselves handling equipment more softly and carefully, as opposed to just turning the volume down on the entire effect (Scruffy, 2019). The absence of music throughout the player's journey over Hyrule is an example of how a decision regarding audio can support a game's rhetorical context. The game developers drew attention to the visual and audio detail in the gameworld by letting the player hear it and prompting them to study it.

While the soundtrack does announce itself in many of the adaptive video game situations, such as combat encounters or entering into a village or town, when left out in the wild, the music will randomly come in and out while maintaining its composition. The randomized nature of 
these needle-drops creates the potential for players to have moments of gameplay scored in novel, unscripted ways, making the game feel more personalized and natural, much like how the radio is utilized in GTA V and Fallout 3. The soundtrack in the wild areas (the vast majority of the map is made up of such places), is scored completely by piano, evoking the softness and delicateness that mirrors the mournful, yet not entirely hopeless, rhetoric of the game. Breath of the Wild employs the function of sound very well, from escalating percussion noises that correspond to how effectively Link hits an enemy, to signaling to the player whether or not the recipe they made would be effective or not (Scruffy, 2019). Whether the player is being prepared for the emergence of the Blood Moon - when enemies become reborn - through the intrusion of a leitmotif, or if they are being rewarded for clearing a camp of enemies by the patented Zelda jingle, the functional use of sound design in Breath of the Wild is constantly assisting the player.

The overworld of Breath of the Wild is full of small details, but the player must seek them out to find them. NPCs do not speak - you can engage in dialogue with them, but it is all written, as opposed to spoken. The gameworld is kept quiet so as not to disrupt to delicate state of the world, always on the precipice of being destroyed forever by the evil force Ganon. The player does get a chance to hear dialogue and experience a sense of community and companionship through the memories that make up one of the (optional) main quests. These flashbacks are the only cinematic moments in the game. The characters (all of whom are since dead or missing) speak out loud with one another, and the player gets to enjoy the relationships Link had with his companions 100 years ago, knowing it all ends tragically for everyone around him. Including spoken dialogue in these scenes is a stark contrast to the quiet the player constantly is surrounded by in the present - the only time dialogue reveals itself again is once the player has beaten the game, freeing the Princess Zelda, who then has a conversation about the future with Link. This 
contrast between two times is established by the audio decisions made by the game developers at Nintendo for Breath of the Wild.

Breath of the Wild often treats music as a reward for the player for completing or finding something specific. As has been touched on briefly above, one of The Legend of Zelda's most recognizable musical phrases is partnered with the revealing of a treasure chest or item. The short, 8-note phrase is immediately recognizable to any Zelda fan. Alongside this phrase, Breath of the Wild has rewarding audio queues for when the player finds collectibles seeds in the world (these are found by solving one of the $900+$ small puzzles integrated into the natural environment, like moving rocks). One of the biggest side-quests in the game, called "Tarrey Town", has a corresponding musical element to reward the player with advancing the quest. On the player's travels, they meet a construction worker who wants a change of scenery and dreams of building his own village. As the player continues to travel the gameworld, they will come across NPCs also unhappy in their surroundings and wanting a change of scenery. The player sends them in the direction of Tarrey Town, and when the player visits the growing town, the song associated with Tarrey Town will add an instrument from the region of the NPC to the composition of the song. When the construction worker is alone, all the player hears when visiting is the bassline of the song. Once the player has populated the town with 5-6 more people, the song becomes filled with different harmonious instruments, mirroring the harmonious nature of the town full of newly immigrated, happy residents. A final example of musical rewards is highlighted by Scruffy (2019) in his video about Breath of the Wild sound design. While gathering footage for the video he was making, Scruffy climbed to the top of a mountain and stood in the stormy weather on the peak. As he stood there recording, the weather suddenly cleared (a rare occurrence on top of a mountain in the game), and the clearing of the weather was 
accompanied by a refreshing leitmotif in the piano composition. Scruffy happened to find this little easter egg by accident, but the finding highlights the importance that music and sound play in The Legend of Zelda series, so much so that the game developers felt the need to include musical rewards in the game, which are as cathartic and satisfying as it is for a player to find a tangible item in the game.

\section{Immersion and Audio}

Video games that have an emphasis on 'play' as opposed to competition tend to have a different relationship to immersion. Games like Super Smash Bros. Ultimate and Call of Duty: Modern Warfare have shorter spurts of gameplay interspersed with periods of set-up. Such sporadic gameplay activity can make it hard for a player to capture a long period of immersion within the game they are playing. In a game like Breath of the Wild, immersion is sought after by the game developers, as the continuous gameplay potential carries with it momentum, something that immersion in a game can help spur on. Andrew Glassner (2004) describes the stages of immersion in video games, saying:

[Level one] begins with curiosity, or the casual desire to know. The next stage is sympathy, in which the player starts to see the world through the eyes of the protagonist. Once the player sees through the protagonist's eyes, the player can identify with the protagonist: seeing elements of the character in him- or herself, and elements of him- or herself in the character. From there it is possible to reach a state of empathy, or emotional bonding with the character. Finally comes a state of transportation, where the player can temporarily lose the boundary between him- or herself and the character. (pp. 81-82)

The final state of this description is aligned with the foundational theory first posited by Mikaly Csikszentmihalyi, which he named flow theory (Csikszentmihalyi, 1990). Breath of the Wild does follow a pattern similar to the one outlined in the block quote above. The immersive elements outlined by Glassner have a tie to emotional empathy. As Nacke and Grimshaw (2011) illustrate, sound can conjure up a significant affective experience that can be a "discrete, conscious, [or] subjective feeling that contributes to, and influences, an individual's emotion" (p. 
265). The pensive environment of the gameworld, accentuated by a delicate score is itself an information-processing system that functions through "the immediate 'gut reaction' or feeling that is triggered by an object, a situation, or even a thought" (Nacke \& Grimshaw, 2008, p. 265). Don Norman (2004) understood information processing through affective means as being just as important as processing information through cognitive means (Norman, 2004, p. 120). Nintendo uses the Breath of the Wild soundtrack to work on the player's emotions, opening up their potential for immersion through affective means. With how important sound and music can be to a player's mood and immersion potential, Nacke and Grimshaw (2008) wonder:

Would it be effective to design an affective real-time sound synthesis sub-engine as part of the game engine where the controllable parameters are not amplitude and frequency but high-level factors such as fear, happiness, arousal, or relaxation? Perhaps these parameters could be governed by the player in the game set-up menu who might opt, for instance, for a more or less emotionally intense experience through the use of a simple fader. (pp. 276-77)

Considering alternative audio possibilities within the same game title in order to prime the player towards the emotional experience they want to have is an interesting and achievable possibility for video games moving forward. Mark Brown (2014) captures the possibility for innovation in the soundscape of video games by noting that music is artificial in gameplay - it is not something a person experiences underscoring their day to day lives. At least, not yet. One could argue that the amount of time that the music in Breath of the Wild fades out and the player is left with only the sounds of nature surrounding Link will stand out to future generations, who constantly have music playing in their ears. Game developers can do anything they want with audio; it is worth considering all of the creative avenues available to developers through the technology available to them.

\section{Super Smash Bros. Ultimate - Audio}

Super Smash Bros. Ultimate does not have the same kind of innovation and meaning behind its music composition and sound design as Breath of the Wild. For Smash, the supporting 
role that audio plays within the overall composition of the game takes two forms. The first is how the sound design of characters is employed to give the characters a sense of personality in order to connect the players with their chosen avatars. The second is how the soundtrack of the game, with its gigantic medley of original Nintendo songs from franchises represented in the game, is a clear celebration of the video games represented within the Smash series. As such, the music soundtrack of the game is often a subject of discussion and debate among the online Smash community.

With the lack of narrative in the game, and the near absence of dialogue amongst the characters, Super Smash Bros. Ultimate has very little opportunity to develop connections between players and characters beyond what is established in their home franchise. While haptic communication does a lot of work towards creating connections between players and characters, game developers rely on sound design as an ample opportunity to further these connections even more. The characters in Super Smash Bros. Ultimate are constantly expressing themselves through sound. Characters often can be heard making cries of pain or triumph as they fight. Many characters, like Captain Falcon and Ness, accompany their signature special attack with a phrase, with Captain Falcon shouting "Falcon Punch" during his Falcon Punch animation, and Ness shouting "PK Fire" during his special attack. These phrases become synonymous with the characters, and sometimes with the game itself, especially for characters like Captain Falcon and Ness, who have been characters in Smash since the original game and Super Smash Bros. Melee, respectively. Ness and Captain Falcon's home franchises are much less widely known than the Earthbound and F-Zero series, making these iterations of the characters the most recognizable to fans. 
The only time Smash characters speak is after a fight has concluded, and the winner is announced. During the post-game animation, the winning character will say one of three phrases. With the lack of dialogue in the game, these phrases become closely tied with the character. Characters who are normally villains in their home franchises, like Meta Knight or Ganondorf will have more insidious phrases, while heroic characters will have more optimistic and triumphant phrases. Players can use these phrases to get a sense of the player's personality, and often the tone of the dialogue matches the villainy of the character. For instance, when Princess Peach, frequent damsel-in-distress of Mario games, wins a fight, she may say 'This is fun!' or 'Peach!' in a sweet, high pitched voice. Ganondorf, on the other hand, celebrates a victory with a deep guttural laugh in a low register that matches the sound design of the character in battle. The same can be said for King K. Rool, the villain of the Donkey Kong series, who also has a low, deep laugh. Meta Knight, a villain in the Kirby franchise does have dialogue in his victory animation, saying things like 'was that even a fight?' in a register equally as low as his villainous counterparts. When the words are few and far between, the tone of the character's sound design in battle and when speaking illustrate their personalities in a simple, yet effective manner for an entertaining fighting game like Smash.

Super Smash Bros. Ultimate has over 24 hours of music to choose from. Where Breath of the Wild decided their soundtrack would be sparse, not containing every classic Zelda song and rearranging many of them, Ultimate decided to go with a 'bigger is better' soundtrack rationale. In a game without a strong narrative, where the majority of the player's time is spent doing battle with friends, strangers, and AI, the game does not need its soundtrack to do the kind of heavy lifting that Breath of the Wild requires from its music. I own Super Smash Bros. Ultimate, and according to the game, I've played it for about 28 hours. I have only heard a fraction of the 
music available to me, as each stage comes with a list of songs from the franchise the stage emulates. The music is also completely customizable in the game menu: the player is able to make playlists, turn off songs they don't like, or mix and match stages from certain franchises with music from other franchises. Super Smash Bros. Ultimate is a game of excess: with 103 stages, 74 characters and counting, and a full day's worth of music, there is no rhetorical message or meaningful moral hidden beneath the surface. Smash is about celebrating Nintendo video games. It's about listening to your favourite Nintendo music, in your favourite Nintendo setting, pitting your favourite Nintendo characters against one another.

If the game has a narrative to it, it is about tapping into the joy of being a child and playing with your toys. In your imagination, Han Solo can fight the Power Rangers in the Batcave. Super Smash Bros. allows players to turn that imagination into an imagined reality. The game has a vibrant competitive online community, but it also is extremely accessible to the player that wants to relax and have fun with friends. The player has the ability to create their own possibility space through the game. They can tinker with the rules, the controls, the characters, the setting, and they can set it to their own music. As Huizinga (1971) says of how play comes to be, "all play moves and has its being within a play-ground marked off beforehand either materially or ideally, deliberately or as a matter of course. [...] All are temporary worlds within the ordinary world, dedicated to the performance of an act apart” (pp. 21-22). Super Smash Bros. Ultimate's soundtrack selection is a great example of how different elements of game design can come together to create the rhetoric of a game, even if that rhetoric is not earth-shattering. 


\section{Conclusion}

Super Smash Bros. Ultimate and Legend of Zelda: Breath of the Wild are microcosms of the kinds of achievement in play that video games can reach. Play should be all-encompassing, but it necessarily has a limit. As Huizinga (1971) writes about the experience of play:

Such at least is the way in which play presents itself to us in the first instance: as an intermezzo, an interlude in our daily lives. As a regularly recurring relaxation, however, it becomes the accompaniment, the complement, in fact an integral part of life in general. It adorns life, amplifies it and is to that extent a necessity both for the individual - as a life function - and for society by reason of the meaning it contains, its significance, its expressive value, its spiritual and social associations, in short, as a culture function. The expression of it satisfies all kinds of communal ideals. It thus has its place in a sphere superior to the strictly biological processes of nutrition, reproduction, and self-preservation. (p. 20)

Video games are wonderful catalysts of play. There are numerous elements of design that go into building a game; there are technical processes that the average player will not even have a sense of within their favourite games. All elements of game design must communicate in some way or another to the player - this is the power of Bogost's (2008) procedural rhetoric. However, some aspects of game design carry more weight than others, in that they are major pathways of communication to the player. Haptics and audio communication are at once complimentary to the rhetoric of a game and the ability to bring a player into a state of play, immersing them either in a single player adventure, or a multiplayer competition. While the player might be immersed in these virtual worlds, they are "no more confinable to virtual worlds than the players are" (Cheng, 2014, p. 14). The sounds of the game and the controller bleed into the real world, “[exemplifying] the medium's materiality writ large, alerting us to possibilities "of being between 'in there' and 'out here': playing at the interface of virtual and visceral experience" (Cheng, 2014, p. 14). Video games remain with the players once they leave the screen. The popularity of online video game communities attests to this fact. Understanding how haptic and 
audio communication can be effectively used to interact with players is hugely important, because it does not remain in the game. One must acknowledge that, "what happens in gameworlds rarely stays in gameworlds - that the sounds and simulations of games can resonate well beyond the glowing screen" (Cheng, 2014, p. 14).

With regards to this paper's research questions, I have shown that audio and haptic communication have an outsized role in the construction and experience of video game play. Whether it be how Breath of the Wild uses a multitude of shortcuts in its control scheme to keep the player immersed in the game, or how mastering the controls in Super Smash Bros. Ultimate can provide players with a better understanding of how to take advantage of frame rate to change the functionality of the game, this paper has illustrated how haptic and audio communication support gameplay design and functionality in video games. The genre of video games can also shift how audio and haptic communication are used. As a fighting game, Ultimate relies on the controller to facilitate the player's understanding of the game, whereas Breath of the Wild's nonroutinized soundtrack perpetuates in the player a sense that the gameworld itself is alive and capable of anything. These communication methods also exhibited their use as devices for the rhetoric of each game, with Ultimate exploring elements of control and identity through the expressive combat system and customizable soundtrack, and Breath of the Wild's exploration of memory, loss, autonomy and the risk of technology through a fragmented soundtrack and a control scheme that ranges from easy use in the wild to structured use in a technologically advanced space that mirrors those same themes.

This paper has sought to bring forward a better understanding of how video game developers have employed and can further employ audio and haptic communication methods in their design philosophy. Ideas of people-at-play have become important philosophical concepts, 
and video games are a modern and popular way in which people are at-play. This paper has investigated how different types of video games rely on audio and haptic communication to support both gameplay elements within the game, and rhetoric espoused by the game. Haptic and audio communication are powerful and effective ways of communicating with video game players. This paper explored how theories of control can be fleshed out, examined, and directly experienced through haptic and audio communication. Video games are maturing into meaningful art and storytelling devices for generations of people. It is crucial that all avenues of how video game rhetoric can be expressed and implemented through game design be explored and considered. There is no limit on the creativity at work in video game play, except for the limits we place upon ourselves. 


\section{Reference List:}

Anderson, R. J., Heath, C. C., Luff, P., \& Moran, T. P. (1993). The social and the cognitive in Human-computer interaction. International Journal: Man-Machine Studies, issue 38. 999-1016. https://doi.org/10.1006/imms.1993.1046

Ash, J. (2012). Technology, technicity, and emerging practices of temporal sensitivity in videogames. Environment and Planning A44. 187-203. https://doi.org/10.1068/a44171

Brown, M. (2016, May 10). Controllers control everything | Game Maker's Toolkit [Video]. YouTube. https://www.youtube.com/watch?v=VJGKDyrR8qc

Baxter, P., Jack, S. (2008, December). Qualitative case study methodology: Study design and implementation for novice researchers. The Qualitative Report, 13(4): 544-559. Retrieved April 16, 2020 from http://www.nova.edu/ssss/QR/QR13-4/baxter.pdf

Cheng, W. (2014). Sound play: video games and the musical imagination. Oxford University Press.

Collins, K. (2008). Game sound: An introduction to the history, theory, and practice of video game music and sound design. MIT Press.

Collins, K. (2013). Playing with sound: A theory of interacting with sound and music in video games. MIT Press.

Concepcion, J., Lindbergh, B. (2016, December 8). 'The Last Guardian' and the video games of the year. The Ringer. https://www.theringer.com/2016/12/8/16047042/the-last-guardianbest-video-games-2016-b304e545bele

Csikszentmihalyi, M. (1990). Flow: The psychology of optimal experience. Harper \& Row.

Garner, A. (2013). Game sound from behind the sofa: an exploration into the fear potential of sound \& psychological approaches to audio-centric, adaptive gameplay. University of Aalborg, 1-254. Retrieved April 23, 2020 from https://vbn.aau.dk/ws/files/316474313/Tom_Garner_13.pdf

Glassner, A. (2004). Interactive storytelling: Techniques for $21_{\text {st }}$ century fiction. Wellesley, Mass.: AK Peters.

Grimshaw, M. (2008). The acoustic ecology of the first-person shooter: The player, sound, and immersion in the first-person shooter computer game. Saarbrücken: VDM Verlag Dr. Mueller

Hamilton, W. A., Garretson, O., \& Kerne, A. (2014). Streaming on Twitch: Fostering participatory communities of play within live mixed media. Computer-Human Interaction Conference '14. 1315-1324. https://doi.org/10.1145/2556288.2557048 
Huizinga, J. (1971). Homo ludens: A study of the play element in culture. Beacon Press.

Klimmt, C., Hartmann, T. (2005, September 23). Effectance, self-efficacy, and the motivation to play video games. Playing Video Games: Motives, Responses, and Consequences. 133145. Retrieved July 10, 2020, from https://www.researchgate.net/publication/284789921_Effectance_selfefficacy_and_the_motivation_to_play_video_games

Klimmt, C., Hartmann, T., Frey, A. (2007, November 6). Effectance and control as determinants of video game enjoyment. CyberPsychology \& Behaviour, 10(6), 845-847. DOI: $10.1089 / \mathrm{cpb} .2007 .9942$

Leino, O. (2019, August). God is a game designer - Accelerating 'Existential Ludology'. DiGRA '19 - Abstract Proceedings of the 2019 DiGRA International Conference: Game, Play, and the Emerging Ludo-Mix. 1-4. Retrieved February 7, 2020, from http://www.digra.org/wp-content/uploads/digital-library/DiGRA_2019_paper_137.pdf

Limperos, A., Schmierbach, M., Kegerise, A., Dardis, F. (2011, June 23). Gaming across different consoles: Exploring the influence of control scheme on game-player enjoyment. Cyberpsychology, Behaviour, and Social Networking, 14(6). https://doiorg.myaccess.library.utoronto.ca/10.1089/cyber.2010.0146

Menon, V., Levitin, D. J. (2005). The rewards of music listening: Response and physiological connectivity of the mesolimbic system. Neuro-Image, 28(1), 175-184.

DOI:10.1016/j.neuroimage.2005.05.053

Miller, K. (2007). Jacking the dial: Radio, race and place in Grand Theft Auto. Ethnomusicology, 51(3), p. 402-438. https://www-jstor-org.ezproxy.lib.ryerson.ca/stable/20174544

Miyamoto, S. (2007, May 9). A creative vision. [Video]. YouTube. https://www.youtube.com/watch?v=En9OXg71ZoE\&pbjreload=101

Nacke, L., Grimshaw, M. (2011). Player-game interaction through affective sound. Games Computing and Creative Technologies: Book Chapters. Paper 3. 264-285. DOI: 10.4018/978-1-61692-828-5.ch013

Norman, D. (2004). Emotional design: why we love (or hate) everyday things. Basic Books.

Norman, D. (2002). The design of everyday things. Basic Books.

Obrist, M., Gatti, E., Maggioni, E., Vi C. T., Velasco, C. (2017). Multisensory experiences in HCI. Institute of Electrical and Electronics Engineers Multimedia Magazine. 9-13. DOI: 10.1109/MMUL.2017.33

Peckham, M. (2017, March 2). Review: The Legend of Zelda: Breath of the Wild is a masterpiece. Time Magazine. https://time.com/4683122/zelda-breath-of-the-wild-review/ 
Pichlmair, M. \& Kayali, F. (2007, January). Levels of sound: On the principles of interactivity in music video games. DiGRA Conference. 424-430. Retrieved February 3, 2020, from http://citeseerx.ist.psu.edu/viewdoc/download?doi=10.1.1.190.2004\&rep=rep1\&type=pdf

Quilitch, H.R., Risley, T. R. (1973). The effects of play materials on social play. Journal of Applied Behaviour Analysis, 6(4), 573-578. DOI:10.1901/jaba.1973.6-573

Ryan, R., Rigby, C. S., Pryzbylski, A. (2006). The motivational pull of video games: A selfdetermination theory approach. Science and Business Media. DOI 10.1007/s11031-0069051-8

Scruffy (2019, July 8). "Invisible” sound design in Breath of the Wild [Video]. YouTube. https://www.youtube.com/watch?time_continue=1\&v=Vgev9Gzybk8

Skalski, P., Tamborini, R., Shelton, A., Buncher, M. (2011, March). Mapping the road to fun: Natural video game controllers, presence, and game enjoyment. New Media \& Society, 13(2), 224-242. DOI: 10.1177/1461444810370949

Suellentrop, C. (2011, October 22). The video-game art of Fumito Ueda. The New Yorker. https://www.newyorker.com/culture/culture-desk/the-video-game-art-of-fumito-ueda

Sutton Smith, B. (1997). The Ambiguity of Play. Harvard University Press.

Urbanek, M, \& Güldenpfennig, F. (2017). Tangible audio game development kit: prototyping audio games with a tangible editor. TEI '17: Proceedings of the Eleventh International Conference on Tangible, Embedded, and Embodied Interaction, 473-479. Retrieved April 27, 2020 from https://doi.org/10.1145/3024969.3025077

Vapidbobcat (2019, September 10). Relaxing Zelda Breath of the Wild Music + Night Ambience Sound [Video]. YouTube. https://www.youtube.com/watch?v=YGaGVajkUjE

Voorhees, G. (2013). Criticism and control: gameplay in the space of possibility. In M. Wysocki (Ed.), Ctrl-alt-play: Essays on control in video gaming. McFarland \& Company Inc. 920.

Wysocki, M. (2013). Ctrl-alt-play: Essays on control in video gaming. McFarland \& Company Inc.

Xue, H., Newman, J., \& Du, J. (2019, July 12). Narratives, identity and community in esports. Leisure Studies. Vol. 38, issue 6. 845-861. https://doi.org/10.1080/02614367.2019.1640778 


\section{Additional Resources:}

Aguado-Delgado, J., Gutiérrez-Martínez, J., Hilera, R. J., de-Marcos, L., \& Otón, S. (2018, August 10). Accessibility in video games: A systematic review. Universal Access in the Information Society. https://doi.org/10.1007/s10209-018-0628-2

Bierre, K., Chetwynd, J., Ellis, B., Hinn, D. M., Ludi, S., \& Westin, T. (2005). Game not over: Accessibility issues in video games. International Conference, Human-Computer Interaction; 2005; Las Vegas, NV. International Conference, Human-Computer Interaction, Las Vegas, NV. Retrieved February 8, 2020, from https://www.researchgate.net/profile/Kevin_Bierre/publication/267403944_Game_Not_O ver_Accessibility_Issues_in_Video_Games/links/546de0d70cf2a7492c560d87.pdf

Brown, M. (2014, November 6). Adaptive soundtracks | designing video game music [Video]. YouTube. https://www.youtube.com/watch?v=b0gvM4q2hdI\&list=PLc38fcMFcV_s7Lf6xbeRfWY Rt7-Vmi_X9\&index $=104 \& \mathrm{t}=0 \mathrm{~s}$

Brown, M. (2017, February 20). The magic of the first Legend of Zelda | Game Maker's Toolkit [Video]. YouTube. https://www.youtube.com/watch?v=KHbQ6ti5BQ0

Iqbal, M. (2020, June 23). Twitch revenue and usage statistics. Business of Apps. https://www.businessofapps.com/data/twitch-statistics/

McLuhan, M. (1994, October). Understanding media: The extensions of man. The MIT Press.

Newzoo (2019, October). 2019 Global games market. [Data set]. 2019 Global Games Market Report. Newzoo. Retrieved February 3, 2020, from https://newzoo.com/key-numbers/

Porter, J. R. (2014, January). Understanding and addressing real-world accessibility issues in mainstream video games. Accessibility and Computing. No. 108. 42-45. https://doi.org/10.1145/2591357.2591364

Statista (2018, June). Number of video gamers worldwide in 2018, by region. [Data set]. Video Games \& Gaming. Statista. Retrieved February 2, 2020, from https://www.statista.com/statistics/293304/number-video-gamers/

TwitchTracker (2020, February). Concurrent channels. [Data set]. Twitch Statistics and Charts. Retrieved July 10, 2020, from https://twitchtracker.com/statistics

TwitchTracker (2020, February). Concurrent viewers. [Data set]. Twitch Statistics and Charts. Retrieved July 10, 2020, from https://twitchtracker.com/statistics 